\title{
INVARIANT REGIONS AND GLOBAL ASYMPTOTIC STABILITY IN AN ISOTHERMAL CATALYST*
}

\author{
JOSÉ M. VEGA
}

\begin{abstract}
A well-known model for the evolution of the (space-dependent) concentration and (lumped) temperature in a porous catalyst is considered. A sequence of invariant regions of the phase space is given, which converges to a globally asymptotically stable region $B$. Quantitative sufficient conditions are obtained for (the region $B$ to consist of only one point and) the problem to have a (unique) globally asymptotically stable steady state.
\end{abstract}

Key words. global stability, invariant regions, porous catalysts, isothermal catalysts

AMS(MOS) subject clessifications. 35B35, 35B40, 35K57, 80A32

1. Introduction. This paper is concerned with a well-known model (Aris [1]) for the evolution of a single reactant concentration $u$ and of the uniform temperature $v$ in an isothermal catalyst

$$
\begin{gathered}
\partial u / \partial t=\Delta u-\phi^{2} f(u, v) \quad \text { in } \Omega, \quad \partial u / \partial n=\sigma(1-u) \quad \text { on } \partial \Omega, \\
d v / d t=\lambda \mu(1-v)+\lambda \phi^{2} \int_{\Omega} f(u, v) d x .
\end{gathered}
$$

Here, $\Delta$ is the Laplacian operator and $n$ is the outward unit normal to the boundary of the bounded domain $\Omega \subset \mathbf{R}^{p}(p=1,2$, or 3$)$. The parameters $\phi^{2}, \sigma, \lambda$, and $\mu$ are strictly positive.

As it has been frequently pointed out in the literature ([1] and references given therein), the isothermal model (1.1), (1.2) is not unrealistic because temperature is often lumped in practice, due to the high conductivity of the solid catalyst. In fact, such a model is a first approximation, as $\beta \rightarrow 0$ and $\nu \rightarrow 0$, of the nonisothermal model, in which temperature is spatially distributed, and given by

$$
L^{-1} \partial v / \partial t=\Delta v+\beta \phi^{2} f(u, v) \quad \text { in } \Omega, \quad \partial v / \partial n=\nu(1-v) \quad \text { on } \partial \Omega .
$$

In this limit, the parameters $\lambda$ and $\mu$ of (1.2) are $\lambda=\beta L / V_{\Omega}$ and $\mu=\nu / S_{\Omega} \beta$, where $V_{\Omega}$ and $S_{\Omega}$ are the volume and the external area of the domain $\Omega$ (see [2]).

The following basic assumptions will be made:

(H.1) The domain $\mathbf{\Omega} \subset \mathbf{R}^{p}$ is bounded and (if $p>1$ ) it is uniformly of class $C^{2+\alpha}$, for some $0<\alpha<1$. Then, it satisfies uniformly the interior and exterior sphere properties: there are two constants, $\rho_{1}>0$ and $\rho_{2}>0$, such that, for every point $q$ of $\partial \Omega$, two hyperspheres, $S_{1}$ and $S_{2}$, of radius $\rho_{1}$ and $\rho_{2}$, are tangent to $\partial \Omega$ at $q$ and satisfy: $S_{1} \subset \Omega$, $\bar{S}_{2} \cap \overline{\mathbf{\Omega}}=\{q\}$.

(H.2) The function $f:\left[0, \infty\left[\times\left[0, \infty\left[\rightarrow \mathbf{R}\right.\right.\right.\right.$ is of class $C^{l}$ and there is a continuous function $F:[0, \infty[\rightarrow \mathbf{R}$ such that: (i) $f(0, v)=0$ for all $v \in[0, \infty[$; (ii) $0<f(u, v) \leqq F(u)$, $\left|f_{t}(u, v)\right| \leqq F(u), 0<f_{v}(u, v)$ for all $\left.(u, v) \in\right] 0, \infty[\times] 0, \infty[$. 
Assumption (H.1) is made for some existence and comparison theorems to be applicable. Assumption (H.2) is satisfied by

$$
\begin{aligned}
f_{1}(u, v) & =u^{m} \exp (\gamma-\gamma / v), \quad m \geqq 1, \quad \gamma \geqq 0, \\
f_{2}(u, v) & =u^{m}(k+u)^{-r} \exp (\gamma-\gamma / v), \quad m \geqq 1, \quad \gamma \geqq 0, \quad k>0, \\
f_{3}(u, v) & =u^{m}\left[k \exp \left(\gamma_{a}-\gamma_{a} / v\right)+u\right]^{-r} \exp (\gamma-\gamma / v), \\
& m \geqq 1, \quad \gamma \geqq r \gamma_{a} \geqq 0, \quad k>0 .
\end{aligned}
$$

The Arrhenius reaction rate function $f_{1}$ is most frequently used to model thermal effects on the reaction rate (see [1]). The Langmuir-Hinshelwood functions $f_{2}$ and $f_{3}$ have received a considerable attention in the literature. Function $f_{2}$ was first proposed to model carbon monoxide oxidation over platinum catalysts, which is the main reaction in automotive pollution-abatement devices. Further experimental evidence showed that several hydrocarbons, such as ethylene and propylene, follow similar rate laws when oxidized over noble metal catalysts (see [3]).

In this paper, some global asymptotic stability properties of the steady state of (1.1), (1.2) will be obtained. Of course, results in the literature for model (1.1), (1.3) (see [4]-[6]) apply to (1.1), (1.2) after small changes; unfortunately they are rather mild: the steady state of $(1.1),(1.3)$ is globally asymptotically stable (and hence, it is unique) if the parameter $\phi^{2}$ is small enough. A slightly stronger result was proven in [5], but it requires the function $f$ to satisfy $f(u, 0)>0$ for $u>0$, and this property does not hold if $f$ is given by (1.4)-(1.6). The results of [4] were obtained by means of a generalized Gronwall inequality. The results of [5], [6] were established by constructing sub- and supersolutions converging to the steady state; the same idea has been used also in the analysis of related reaction-diffusion problems (see, e.g., [7]-[10]).

Our approach is somewhat different, although it is also based on comparison theorems. We shall construct a sequence $\left\{B_{m}\right\}$ of invariant, stable regions of the phase space of $(1.1),(1.2)$, such that every region $B_{m}$ traps the transient state of the system in a finite time for arbitrary initial conditions. If the sequence $\left\{B_{m}\right\}$ converges to a region of the phase space $B$ in an appropriate uniform sense, then such region is globally asymptotically stable for (1.1), (1.2). Therefore, $B$ contains the nonwandering set of (1.1), (1.2) (i.e., the set of points $(u, v)$ of the phase space of (1.1), (1.2) such that, for every neighborhood of $(u, v), U \subset C(\bar{\Omega}) \times \mathbf{R}$, and every $T>0$, there are a constant $t>T$ and a point $\left(u_{0}, v_{0}\right) \in U$ that are such that the solution of (1.1), (1.2), with initial conditions $(u(0), v(0))=\left(u_{0}, v_{0}\right)$, satisfies $(u(t), v(t)) \in U$ (see Hirsch [11])). In particular, $B$ contains every (stable or unstable) steady state, periodic, or quasiperiodic solution, $\cdots$, of (1.1), (1.2). If $B$ consists of only one point, then such point is a globally asymptotically stable (and hence, a unique) steady state of (1.1), (1.2). This method of finding globally asymptotically stable invariant regions for nonmonotone flows is similar to that used by Leung [12] in his study of some prey-predator problems; in some sense, the ideas are in the spirit of the work by Keller [13] and Sattinger [14] on semilinear elliptic problems.

In $\S 2$ we shall prove some basic results and state some definitions. In $\$ 3$, a sequence of invariant regions of the phase space of $(1.1),(1.2)$, of the type described above, will be obtained. The results of $\S 3$ will be applied in $\S 4$, to obtain some quantitative sufficient conditions for the steady state of (1.1), (1.2) to be globally asymptotically stable for a function $f$ of a rather general type: $f(u, v)=$ $g(u) \exp (\gamma-\gamma / v)$, which includes the particular instances of (1.4) and (1.5). In particular, we shall obtain global asymptotic stability of the steady state if $\phi^{2}$ is 
sufficiently small or large, or if the function $g$ is increasing and $\gamma$ is sufficiently small. As a corollary, some sufficient conditions for the steady state of (1.1), (1.2) to be unique will be obtained. For results on existence and uniqueness of the steady state of (1.1), (1.3), see [4], [6], [15], [16]. It should be pointed out that to prove uniqueness for large $\phi^{2}$ is not an easy task (see [16]).

The following notation will be widely used in the sequel. If $\Omega$ is defined as above, and if $u_{1}, u_{2} \in C(\bar{\Omega})$, then $u_{1} \leqq u_{2}$ will mean that $u_{1}(x) \leqq u_{2}(x)$ for all $x \in \bar{\Omega}$, and $u_{1}<u_{2}$ will mean that $u_{1} \leqq u_{2}$ and $u_{1} \neq u_{2}$. If $u_{1}(x)<u_{2}(x)$ for all $x \in \bar{\Omega}$, then we shall write $u_{1} \ll u_{2}$.

2. Preliminary results and definitions. Let us first consider some basic results concerning the evolution problem (1.1), (1.2), with initial conditions

$$
u(x, 0)=\tilde{u}(x) \geqslant 0 \text { for all } x \in \bar{\Omega}, \quad v(0)=\tilde{v} \geqq 0,
$$

where $\tilde{u} \in C^{2}(\bar{\Omega})$ and satisfies the boundary condition (1.1). By a (classical) regular solution of (1.1), (1.2), (2.1) we shall mean a couple of functions

$$
u \in C^{1,0}\left(\bar{\Omega} \times\left[0, \infty[) \cap C^{2,1}(\bar{\Omega} \times] 0, \infty[), \quad v \in C^{1}([0, \infty[),\right.\right.
$$

which satisfy $(1.1),(1.2),(2.1)$ pointwise, and are such that $u(\cdot, t) \geqq 0, v(t) \geqq 0$ for all $t>0$. Here, $u \in C^{1,0}$ means that the functions $(x, t) \rightarrow u$ and $(x, t) \rightarrow D u$ are continuous. $u \in C^{2,1}$ means that $u \in C^{1,0}$ and the functions $(x, t) \rightarrow D^{2} u$ and $(x, t) \rightarrow \partial u / \partial t$ are continuous, where $D u$ and $D^{2} u$ are the matrices of first- and second-order $x$-derivatives of $u$. Observe that negative concentrations and temperatures are not allowed since they do not make sense from the physical point of view. sequel.

The following consequence of maximum principles will be widely used in the

LEMMA 2.1. Let $\Omega$ be as in assumption (H.1), and let $W$ be a function of $C^{1,0}(\bar{\Omega} \times$ $\left[0, \infty[) \cap C^{2,1}(\bar{\Omega} \times] 0, \infty[)\right.$, such that

(a) $W(x, 0) \geqq 0$ for all $x \in \bar{\Omega}$.

(b) $\partial W / \partial t>\Delta W$ for all $(x, t) \in \Omega \times] 0, \infty[$ such that $W(x, t)<0$.

(c) $\partial W / \partial n>0$ for all $(x, t) \in \partial \Omega \times] 0, \infty[$ such that $W(x, t)<0$.

Then $W(x, t) \geqq 0$ for all $(x, t) \in \bar{\Omega} \times[0, \infty[$.

Proof. The result follows by standard arguments, using maximum principles (Protter and Weinberger [17]).

Global existence and uniqueness of solution of (1.1), (1.2), (2.1) will be a consequence of the following a priori bound.

LeMmA 2.2. Under assumptions (H.1) and (H.2), let $u=u(x, t), v=v(t)$, be a regular solution of (1.1), (1.2), (2.1). Then, there is a constant $\alpha>0$ and a function $\psi \in C^{2}(\bar{\Omega})$, such that

$$
u(x, t) \leqq 1+\psi(x) \exp (-\alpha t) \text { for all }(x, t) \in \bar{\Omega} \times[0, \infty[.
$$

Proof. As is well known, the problem

$$
\Delta \psi+\alpha \psi=0 \quad \text { in } \Omega, \quad \partial \psi / \partial n+\sigma \psi=0 \quad \text { on } \partial \Omega,
$$

has a smallest eigenvalue $\alpha>0$, and eigenfunctions $\psi$ such that $\psi \gg 0$. Hence, $\psi$ may be chosen to be such that $\psi \geqq 2(\tilde{u}-1), \psi \gg 0$ and the function $W=W(x, t)=$ $1-u(x, t)+[2 \exp (-\alpha t)-\exp (-2 \alpha t)] \psi(x) / 2$ satisfies $W(\cdot, t) \geqq 0$ for all $t \geqq 0$, as it comes out when Lemma 2.1 is applied. 
THEOREM 2.3. Under assumptions (H.1) and (H.2), the problem (1.1), (1.2), (2.1) has a unique regular solution if $\tilde{u} \in C^{2}(\bar{\Omega})$ and $\tilde{u}$ satisfies the boundary condition.

Proof. For given $\tilde{u}$ and $\tilde{v}$, let $\psi$ be as in Lemma 2.2 and let $k=1+\max \{\psi(x): x \in \bar{\Omega}\}$. Then, no regular solution of (1.1), (1.2), (2.1) is affected when $f$ is replaced, in (1.1), (1.2), by another function, $\tilde{f}: \mathbf{R}^{2} \rightarrow \mathbf{R}$, that is defined by: $\tilde{f}(u, v)=0$ for $u<0, \hat{f}(u, v)=$ $f(u,|v|)$ for $0 \leqq u \leqq k, f(u, v)=f(k,|v|)$ for $u>k$. Any solution of (1.1), (1.2), (2.1), with $f$ modified as above, is a regular solution of the original problem (the converse is trivially satisfied). That is, if $u \in C^{\mathbf{l . 0}}\left(\bar{\Omega} \times\left[0, \infty[) \cap C^{2,1}(\bar{\Omega}] 0,, \infty[), v \in C^{1}([0, \infty[)\right.\right.$ is a solution of the modified problem, then $u(\cdot, t) \geqq 0$ and $v(t) \geqq 0$ for all $t>0$. Since $\tilde{f}(u, v) \geqq 0$ for all $(u, v) \in \mathbf{R}^{2},(1.2)$ yields $d v / d t \geqq \lambda \mu(1-v)$ and $v(t) \geqq 0$ for all $t>0$; also, $u(\cdot, t) \geqq 0$ for all $t>0$, as it comes out when Lemma 2.1 is applied to $W=$ $u \exp (-t)$, and it is taken into account that $\tilde{f}(u, v)=0$ for $u<0$. Then, we only need to prove the conclusion of the theorem when $f$ is replaced by $\tilde{f}$, and this comes out from standard theory on semilinear equations (e.g., from [18, Cor. 3.3.5] and [19, Lemma 4.2]), when taking into account that $\tilde{f}$ is locally Lipschitz and globally bounded.

The following $\varepsilon-\delta$ stability definitions of the Lyapunov type will be used in the sequel. They are given in terms of the distance $d$, associated with the norm

$$
\|(u, v)\|=\max \{|u(x)|: x \in \bar{\Omega}\}+|v| \quad \text { for }(u, v) \in C(\bar{\Omega}) \times \mathbf{R} .
$$

The distance between $(u, v) \in C(\bar{\Omega}) \times \mathbf{R}$ and $B \subset C(\overline{\mathbf{\Omega}}) \times \mathbf{R}$ is defined as usually $d[(u, v), B]=\inf \left\{\left\|\left(u-u^{\prime}, v-v^{\prime}\right)\right\|:\left(u^{\prime}, v^{\prime}\right) \in B\right\}$. Observe that $C(\bar{\Omega}) \times \mathbf{R}$ includes the phase space of (1.1), (1.2), (2.1).

DEFINITION 2.4. Let $B \subset C(\bar{\Omega}) \times \mathbf{R}$. $B$ is said to be an invariant region for the problem $(1.1),(1.2),(2.1)$ if, for any regular solution of the problem, $(u(\cdot, 0), v(0)) \in B$ implies $(u(\cdot, t), v(t)) \in B$ for all $t>0$. An invariant region $B$ is said to be stable if, for every $\varepsilon>0$, there is a $\delta>0$ such that for every regular solution of the problem $d[(u(\cdot, 0), v(0)), B]<\delta$ implies $d[(u(\cdot, t), v(t)), B]<\varepsilon$ for all $t>0$. A region $B$ is said to be globally asymptotically attracting if every regular solution of the problem satisfies $d[(u(\cdot, t), v(t)), B] \rightarrow 0$ as $t \rightarrow \infty$. An invariant region $B$ is said to be globally asymptotically stable if it is stable and globally asymptotically attracting. A region $B$ is said to be globally finitely attracting if, for every regular solution of the problem, there is a constant $T<\infty$ such that $(u(\cdot, t), v(t)) \in B$ for all $t \geqq T$.

The concept of globally finitely attracting region and the following lemma will be used in $\S 3$.

LEMMA 2.5. Let the sequence of regions $\left\{B_{m}\right\}$ and the region $B=\cap\left\{B_{m}: m \in N\right\}$, of $C(\bar{\Omega}) \times \mathbf{R}$, be such that

(a) Every $B_{m}$ is invariant, and globally finitely attracting for (1.1), (1.2), (2.1);

(b) For every $m \in \mathbf{N}$, there are two constants, $\varepsilon_{m}>0$ and $\delta_{m}>0$, such that $N\left(B, \delta_{m}\right) \subset B_{m} \subset N\left(B, \varepsilon_{m}\right)$, where

$$
N(B, \delta)=\{(u, v) \in C(\tilde{\Omega}) \times \mathbf{R}: d[(u, v), B]<\delta\} ;
$$

(c) $\varepsilon_{m} \rightarrow 0$ as $m \rightarrow \infty$.

Then the region $B$ is invariant and globally asymptotically stable for the problem.

Proof. Since $B=\cap\left\{B_{m}: m \in \mathrm{N}\right\}$, the region $B$ is clearly invariant. $B$ is stable since for every $\varepsilon>0$ there is an $m \in \mathbf{N}$ such that $\varepsilon_{m}<\varepsilon$; then the definition of stable region is satisfied with $\delta=\delta_{m}$. Finally, $B$ is globally asymptotically attracting since for every $\varepsilon>0$ there is a constant $T$ such that $d[(u(\cdot, t), v(t)), B]<\varepsilon$ for all $t \geqslant T$. To see that, take $m$ such that $\varepsilon_{m}<\varepsilon$ and take into account that $B_{m}$ is globally finitely attracting and $B_{m} \subset N\left(B, \varepsilon_{m}\right) \subset N(B, \varepsilon)$. 
3. Invariant regions. In this section, we obtain a sequence of regions satisfying the hypothesis of Lemma 2.5 , which leads to a globally asymptotically stable region of the phase space of (1.1), (1.2), (2.1).

Let $\left\{\alpha^{m}\right\}$ be a strictly decreasing sequence of real numbers, such that $\alpha^{m} \rightarrow 1$ as $m \rightarrow \infty$ and $\alpha^{0}\left(\alpha^{1}-1\right) \leqq M^{-1} \alpha^{1} f\left(\alpha^{0}, 1 / \alpha^{0}\right)$, where the constant $M>0$ is defined below. Let the sequence $\left\{\alpha_{m}\right\}$ be defined by $\alpha_{m}=1 / \alpha^{m}$ for all $m \in N$. From assumption (H.2) (see Introduction), it turns out that there is a constant $M>0$ and a function $h:\left[0, \alpha^{0}\right] \times$ $\left[\alpha_{0}, \infty\left[\rightarrow \mathbf{R}\right.\right.$, of class $C^{1}$ and bounded, such that $h \geqq 0, \partial h / \partial u \geqq 0, \partial h / \partial v \geqq 0,-\partial h / \partial u \leqq$ $\partial f / \partial u<M$ for all $(u, v) \in\left[0, \alpha^{0}\right] \times\left[\alpha_{0}, \infty[\right.$.

We consider the sequence of regions $\left\{B_{m}\right\} \subset C(\bar{\Omega}) \times \mathbf{R}$, defined by

$$
B_{m}=\left\{(u, v) \in C(\overline{\mathbf{\Omega}}) \times \mathbf{R}: u_{m} \leqq u \leqq u^{m}, G_{m} \leqq v+\lambda \int_{\Omega} u d x \leqq G^{m}, v_{m} \leqq v \leqq v^{m}\right\},
$$

where $u_{0}, u^{0}, G_{0}, G^{0}, v_{0}$, and $v^{0}$ are

$$
\begin{gathered}
u_{0}=0, \quad G_{0}=\alpha_{0}-\mu^{-1} \sigma S_{\Omega}\left(\alpha^{0}-1\right), \quad v_{0}=\alpha_{0}, \quad u^{0}=\alpha^{0}, \\
G^{0}=\alpha^{0}\left(1+\lambda V_{\Omega}\right)+\mu^{-1} \sigma S_{\Omega}, \\
v^{0}=\alpha^{0}+\mu^{-1} \phi^{2} V_{\Omega} \sup \left\{f\left(\alpha^{0}, v\right)+h\left(\alpha^{0}, v\right): v \geqq \alpha_{0}\right\}
\end{gathered}
$$

( $V_{\Omega}$ and $S_{\Omega}$ are the volume of $\Omega$ and the area of $\partial \Omega$, respectively), and where $u_{m}, u^{m}$, $G_{m}, G^{m}, v_{m}$, and $v^{m}(m \geqq 1)$ are defined, inductively, by

$$
\begin{gathered}
\Delta u_{m}-\phi^{2} M u_{m}=\alpha_{m} \phi^{2}\left[f\left(u_{m-1}, v^{m-1}\right)-M u_{m-1}\right] \quad \text { in } \Omega \\
\partial u_{m} / \partial n=\sigma\left(\alpha_{m}-u_{m}\right) \text { on } \partial \Omega, \\
\Delta u^{m}-\phi^{2} M u^{m}=\alpha^{m} \phi^{2}\left[f\left(u^{m-1}, v_{m-1}\right)-M u^{m-1}\right] \text { in } \Omega, \\
\partial u^{m} / \partial n=\sigma\left(\alpha^{m}-u^{m}\right) \text { on } \partial \Omega, \\
G_{m}=\alpha_{m}+\lambda \int_{\Omega} u_{m} d x+\mu^{-1} \sigma \int_{\partial \Omega}\left(1-u^{m}\right) d s \\
G^{m}=\alpha^{m}+\lambda \int_{\Omega} u^{m} d x+\mu^{-1} \sigma \int_{\partial \Omega}\left(1-u_{m}\right) d s \\
v_{m}=\max \left\{\alpha_{m}, G_{m}-\lambda \int_{\Omega} u^{m} d x, w_{m}\right\}, \quad v^{m}=\min \left\{G^{m}-\lambda \int_{\Omega} u_{m} d x, w^{m}\right\}
\end{gathered}
$$

with

$$
\begin{aligned}
& w_{m}=\alpha_{m}+\mu^{-1} \phi^{2} \int_{\Omega}\left[f\left(u_{m}, v_{m-1}\right)+h\left(u_{m}, v_{m-1}\right)-h\left(u^{m}, v^{m-1}\right)\right] d x, \\
& w^{m}=\alpha^{m}+\mu^{-1} \phi^{2} \int_{\Omega}\left[f\left(u^{m}, v^{m-1}\right)+h\left(u^{m}, v^{m-1}\right)-h\left(u_{m}, v_{m-1}\right)\right] d x .
\end{aligned}
$$

LeMMA 3.1. Let $m \geqq 0$ be an integer. If a regular solution of (1.1), (1.2), (2.1) satisfies, for all $t \geqq 0$,

$$
\begin{gathered}
u_{0} \leqq u(\cdot, t) \leqq u^{0}, \quad \alpha_{0} \leqq v(t) \quad \text { if } m=0, \quad \text { or } \\
u_{m} \leqq u(\cdot, t) \leqq u^{m}, \quad v_{m-1} \leqq v(t) \leqq v^{m-1} \quad \text { if } m \geqq 1,
\end{gathered}
$$


then there is a constant $T$ such that for all $t \geq T$,

$$
G_{m} \leqq v(t)+\lambda \int_{\Omega} u(x, t) d x \leqq G^{m}, \quad v_{m} \leqq v(t) \leqq v^{m}
$$

If, in addition, the inequalities (3.12) hold for $t=0$, then they also hold for all $t>0$.

Proof. By using (3.10) or (3.11), the time derivative of $G(t)=v(t)+\lambda \int_{\Omega} u(x, t) d x$,

$$
d G / d t=\lambda \mu(1-G)+\lambda^{2} \mu \int_{\Omega} u d x+\lambda \sigma \int_{\partial \Omega}(1-u) d s,
$$

and $d v / d t$ are easily seen to satisfy, for all $t \geq 0$,

$$
\begin{aligned}
& \lambda \mu\left(G_{m}-G+1-\alpha_{m}\right) \leqq d G / d t \leqq \lambda \mu\left(G^{m}-G+1-\alpha^{m}\right) \quad \text { for } m \geqq 0, \\
& \lambda \mu(1-v) \leqq d v / d t \leqq \lambda \mu\left(v^{0}-v+1-\alpha^{0}\right), \\
& \lambda \mu\left(w_{m}-v+1-\alpha_{m}\right) \leqq d v / d t \leqq \lambda \mu\left(w^{m}-v+1-\alpha^{m}\right) \quad \text { for } m \geqq 1 .
\end{aligned}
$$

From these inequalities, the conclusion of the lemma readily follows.

LEMMA 3.2. The sequences defined by (3.1)-(3.7) satisfy, for all $m \in \mathbf{N}$ :

A. $u_{m} \ll u_{m+1} \ll u^{m+1} \ll u^{m}, G_{m}<G_{m+1}<G^{m+1}<G^{m}, v_{m}<v_{m+1}<v^{m+1}<v^{m}$.

B. $B_{m}$ is an invariant region for the problem (1.1), (1.2), (2.1).

C. $B_{m}$ is a globally, finitely attracting region for (1.1), (1.2), (2.1).

Proof. An induction argument will be used in the three cases. It will be proved that the required property holds for $m=0$ and that it is satisfied for $m=p$ if it holds for $m=p-1$.

A. Both steps of the induction argument are easily accomplished by means of maximum principles.

B. To prove that $B_{0}$ is invariant, observe that if a regular solution of (1.1), (1.2), (2.1) is such that $(u(\cdot, 0), v(0)) \in B_{0}$, then it satisfies, for all $t \geqq 0:(i) u(\cdot, t) \geqq u_{0}=0$ (definition of regular solution); (ii) $v(t) \geqq v_{0}=\alpha_{0}$ (use the inequality $d v / d t \geqq$ $\lambda \mu(1-v)$ ); (iii) $u(\cdot, t) \leqq u^{0}=\alpha^{0}$ (apply Lemma 2.1 with $W=\alpha^{0}-u$ ); and (iv) $G_{0} \leqq$ $v(t)+\lambda \int_{\Omega} u(x, t) d x \leqq G^{0}, v(t) \leqq v^{0}$ (Lemma 3.1). In the same way, if $B_{p-t}$ is invariant and if $(u(\cdot, 0), v(0)) \in B_{p} \subset B_{p-1}$, then for all $t \geqq 0,(u(\cdot, t), v(t)) \in B_{p-1}$ and (i) $u_{p} \leqq$ $u(\cdot, t) \leqq u^{p}$ (apply Lemma 2.1 with $W=u-u_{p}$ and with $W=u^{p}-u$ ), and (ii) $G_{p} \subseteq$ $v(t)+\lambda \int_{\Omega} u(x, t) d x \leqq G^{p}, v_{p} \leqq v(t) \leqq v^{p}$ (Lemma 3.1).

C. To prove that $B_{0}$ is globally finitely attracting, observe that any regular solution of (1.1), (1.2), (2.1) satisfies, for some finite constants, $T_{1}, T_{2}$, and $T_{3}$ (i) $0=u_{0} \leqq$ $u(\cdot, t) \leqq u^{0}=\alpha^{0}$ for all $t \geqq T_{1}$ (Lemma 2.2); (ii) $v(t) \geqq v_{0}=\alpha_{0}$ for all $t \geqq T_{2}$ (use the inequality $d v / d t \geqq \lambda \mu(1-v)$ ); and (iii) $G_{0} \leqq v(t)+\lambda \int_{\Omega} u(x, t) d x \leqq G^{0}, v_{0} \leqq v(t) \leqq v^{0}$ for all $t \geqq T_{3}$ (take the time variable $t=t-\max \left\{T_{1}, T_{2}\right\}$ and apply Lemma 3.1).

Now, we assume that $B_{p-1}$ satisfies property $C$ and prove that $B_{p}$ also satisfies it. Let $(u, v)$ be a regular solution of (1.1), (1.2), (2.1). By taking an appropriate origin of the time scale, we may assume that $u_{p-1} \leqq u(\cdot, t) \leqq u^{p-1}$ and $v_{p-1} \leqq v(t) \leqq v^{p-1}$ for all $t \geqq 0$. Then there are finite constants, $T_{1}$ and $T_{2}$, such that (i) $u_{p} \leqq u(\cdot, t) \leqq u^{p}$ for all $t \geqq T_{1}$ (apply Lemma 2.1 with $W=u+\psi_{p} \exp (-\alpha t)-\alpha^{p} u_{p}$ and with $W=\alpha_{p} u^{p}+$ $\psi^{p} \exp (-\alpha t)-u$, where $\alpha>0$ is the smallest eigenvalue of $(2.2)$, and $\psi_{p} \geqq 0$ and $\psi^{p} \geqq 0$ are eigenfunctions such that $\psi_{p} \geqq \alpha^{p} u_{p}-u(\cdot, 0)$ and $\psi^{p} \geqq u(\cdot, 0)-\alpha_{p} u^{p}$ ); (ii) $G_{p} \leqq$ $v(t)+\lambda \int_{\Omega} u(x, t) d x \leqq G^{p}, v_{p} \leqq v(t) \leqq v^{p}$ for all $t \geqq T_{2}$ (take the time variable $t=t-T_{1}$ and apply Lemma 3.1 ). 
THEOREM 3.3. A. The sequences defined by (3.2)-(3.7) satisfy $u_{m} \rightarrow u_{*}, u^{m} \rightarrow u^{*}$, uniformly in $\bar{\Omega} ; G_{m} \rightarrow G_{*}, G^{m} \rightarrow G^{*}, v_{m} \rightarrow v_{*}, v^{m} \rightarrow v^{*}$, as $m \rightarrow \infty$, where $u_{*}, u^{*} \in C^{2}(\bar{\Omega})$, $G_{*}, G^{*}, v_{*}$, and $v^{*}$ satisfy

$$
\begin{aligned}
& \Delta u_{*}=\phi^{2} f\left(u_{*}, v^{*}\right) \quad \text { in } \Omega, \quad \partial u_{*} / \partial n=\sigma\left(1-u_{*}\right) \quad \text { on } \partial \Omega, \\
& \Delta u^{*}=\phi^{2} f\left(u^{*}, v_{*}\right) \quad \text { in } \Omega, \quad \partial u^{*} / \partial n=\sigma\left(1-u^{*}\right) \quad \text { on } \partial \Omega, \\
& G_{*}=1+\lambda \int_{\Omega} u_{*} d x+\mu^{-1} \sigma \int_{\partial \Omega}\left(1-u^{*}\right) d s, \\
& G^{*}=1+\lambda \int_{\Omega} u^{*} d x+\mu^{-1} \sigma \int_{\partial \Omega}\left(1-u_{*}\right) d s, \\
& v_{*}=\max \left\{1, G_{*}-\lambda \int_{\Omega} u^{*} d x,\right. \\
& \left.\quad 1+\mu^{-1} \phi^{2} \int_{\Omega}\left[f\left(u_{*}, v_{*}\right)+h\left(u_{*}, v_{*}\right)-h\left(u^{*}, v^{*}\right)\right] d x\right\} \\
& v^{*}=\min \left\{G^{*}-\lambda \int_{\Omega} u_{*} d x,\right. \\
& \left.\quad 1+\mu^{-1} \phi^{2} \int_{\Omega}\left[f\left(u^{*}, v^{*}\right)+h\left(u^{*}, v^{*}\right)-h\left(u_{*}, v_{*}\right)\right] d x\right\} \\
& 0 \ll u_{*} \leqq u^{*} \ll 1, \quad 1 \leqq G_{*} \leqq G^{*}<\infty, \quad 1 \leqq v_{*} \leqq v^{*}<\infty
\end{aligned}
$$

B. The region

$$
\boldsymbol{B}=\left\{(u, v) \in C(\overline{\mathbf{\Omega}}) \times \mathbf{R}: u_{*} \leqq u \leqq u^{*}, G_{*} \leqq v+\lambda \int_{\Omega} u d x \leqq G^{*}, v_{*} \leqq v \leqq v^{*}\right\}
$$

is invariant, and globally asymptotically stable for the problem (1.1), (1.2), (2.1).

Proof. A. The monotone, bounded sequences $\left\{G_{m}\right\},\left\{G^{m}\right\},\left\{v_{m}\right\}$, and $\left\{v^{m}\right\}$ are convergent, and their limits satisfy (3.18) (Lemma 3.2A). In the same way, the monotone, bounded sequences $\left\{u_{m}\right\}$ and $\left\{u^{m}\right\}$ are pointwise-convergent to some functions $u_{*}$ and $u^{*}$ satisfying $0 \ll u_{*} \leqq u^{*}$. By means of elliptic estimates, it may be seen that $u_{*}$ and $u^{*}$ are twice continuously differentiable and satisfy (3.13), (3.14), and that the convergence is uniform in $\bar{\Omega}$ (only slight modifications are necessary in the proof of Theorem 2.1 of [14], or in the proof of Theorem 10.3 of [20]). Then (3.15)-(3.17) are obtained as limits of (3.6), (3.7). The inequality $u^{*} \ll 1$ is easily obtained when maximum principles are applied to (3.14).

B. The sequence $\left\{B_{m}\right\}$ satisfies the hypothesis (a) of Lemma 2.5 (Lemma 3.2). Hypothesis (c) is also satisfied if $\delta_{m}$ and $\varepsilon_{m}$ are

$$
\begin{array}{r}
\left(1+\lambda V_{\Omega}\right) \delta_{m}=\min \left\{\min \left\{u_{*}-u_{m}: x \in \bar{\Omega}\right\}, \min \left\{u^{m}-u^{*}: x \in \bar{\Omega}\right\},\right. \\
\left.G_{*}-G_{m}, G^{m}-G^{*}, v_{*}-v_{m}, v^{m}-v^{*}\right\}, \\
\varepsilon_{m}=3\left(1+\lambda V_{\Omega}\right) \max \left\{\max \left\{u_{*}-u_{m}: x \in \bar{\Omega}\right\}, \max \left\{u^{m}-u^{*}: x \in \bar{\Omega}\right\},\right. \\
\left.G_{*}-G_{m}, G^{m}-G^{*}, v_{*}-v_{m}, v^{m}-v^{*}\right\},
\end{array}
$$

Observe that $\delta_{m}>0$ for $m=0,1, \cdots$, as it comes out from the inequalities $u_{m} \ll u_{*} \leqq$ $u^{*} \ll u^{m}, G_{m}<G_{*} \leqq G^{*}<G^{m}, v_{m}<v_{*} \leqq v^{*}<v^{m}$ (for $m=0,1, \cdots$ ), which are easily obtained from Lemma 3.2A. 
Then, we only need to prove that hypothesis (b) is also satisfied. To this end, observe that.

(i) If $(u, v) \in N\left(B, \delta_{m}\right)$, then there is $\left(u^{\prime}, v^{\prime}\right) \in B$ such that

$$
d\left[(u, v),\left(u^{\prime}, v^{\prime}\right)\right]=\max \left\{\left|u-u^{\prime}\right|: x \in \bar{\Omega}\right\}+\left|v-v^{\prime}\right|<\delta_{m} .
$$

Hence, $u_{*} \leqq u^{\prime} \leqq u^{*}, G_{*} \leqq v^{\prime}+\lambda \int_{\Omega} u^{\prime} d x \leqq G^{*}, v_{*} \leqq v^{\prime} \leqq v^{*}$ and

$$
\begin{gathered}
u^{m}-u \geqq\left(u^{m}-u^{*}\right)+\left(u^{*}-u^{\prime}\right)-\left|u-u^{\prime}\right| \geqq\left(1+\lambda V_{\Omega}\right) \delta_{m}+0-\delta_{m} \geqq 0, \\
G^{m}-v-\lambda \int_{\Omega} u d x \geqq\left(G^{m}-G^{*}\right)+\left(G^{*}-v^{\prime}-\lambda \int_{\Omega} u^{\prime} d x\right) \\
-\left(\left|v-v^{\prime}\right|+\lambda \int_{\Omega}\left|u-u^{\prime}\right| d x\right) \\
\geqq\left(1+\lambda V_{\Omega}\right) \delta_{m}+0-\left(1+\lambda V_{\Omega}\right) \delta_{m} \geqq 0, \\
v^{m}-v \geqq\left(v^{m}-v^{*}\right)+\left(v^{*}-v^{\prime}\right)-\left|v-v^{\prime}\right| \geqq\left(1+\lambda V_{\Omega}\right) \delta_{m}+0-\delta_{m} \geqq 0 .
\end{gathered}
$$

Similarly, it is easily seen that $u-u_{m} \geqq 0, v+\lambda \int_{\Omega} u d x-G_{m} \geqq 0$ and $v-v_{m} \geqq 0$. Hence, $(u, v) \in B_{m}$.

(ii) If $(u, v) \in B_{m}$ and if $\left(u^{\prime}, v^{\prime}\right) \in C(\bar{\Omega}) \times \mathbf{R}$ is given by

$$
\begin{aligned}
& u^{\prime}(x)=\max \left\{u_{*}(x), \min \left\{u(x), u^{*}(x)\right\}\right\} \text { for } x \in \bar{\Omega} \\
& v^{\prime}=\max \left\{v_{*}, G_{*}-\lambda \int_{\Omega} u^{\prime} d x, \min \left\{v, v^{*}, G^{*}-\lambda \int_{\Omega} u^{\prime} d x\right\}\right\},
\end{aligned}
$$

then $\left(u^{\prime}, v^{\prime}\right) \in B$ and $d\left[(u, v),\left(u^{\prime}, v^{\prime}\right)\right] \leqq 2 \varepsilon_{m} / 3<\varepsilon_{m}$, as is easily seen. Therefore, $(u, v) \in$ $N\left(B, \varepsilon_{m}\right)$.

Remark 3.4. Some remarks about the results above are in order.

A. It is easily seen, by means of an induction argument, that for every solution of (3.13)-(3.18), $\left(u_{*}, u^{*}, G_{*}, G^{*}, v_{*}, v^{*}\right)$, the sequence defined by (3.2)-(3.7) satisfies

$$
u_{m} \ll u_{*} \leqq u^{*} \ll u^{m}, \quad G_{m}<G_{*} \leqq G^{*}<G^{m}, \quad v_{m}<v_{*} \leqq v^{*}<v^{m},
$$

for all $m \in \mathbf{N}$. Therefore, the solution of (3.13)-(3.18) that is approached as $m \rightarrow \infty$ by the sequence (3.2)-(3.7), $\left(\tilde{u}_{*}, \tilde{u}^{*}, \tilde{G}_{*}, \tilde{G}^{*}, \tilde{v}_{*}, \tilde{v}^{*}\right)$, is maximal in the following sense: any other solution of (3.13)-(3.18) is such that $\tilde{u}_{*} \leqq u_{*} \leqq u^{*} \leqq \tilde{u}^{*}, \tilde{G}_{*} \leqq G_{*} \leqq G^{*} \leqq \tilde{G}^{*}$, $\tilde{v}_{*} \leqq v_{*} \leqq v^{*} \leqq \tilde{v}^{*}$. Since such maximal solution of (3.13)-(3.18) is necessarily unique, the region $B$ of Theorem 3.3 is independent of the choice of the sequence $\left\{\alpha^{m}\right\}$ and of the constant $M$. Furthermore, if one takes $\alpha_{m}=\alpha^{m}=1$ for all $m \in N$ in (3.1)-(3.9), the following sequence of regions is obtained

$$
B_{m}=\left\{(u, v) \in C(\bar{\Omega}) \times \mathbf{R}: u_{m} \leqq u \leqq u^{m}, G_{m} \leqq v+\lambda \int_{\Omega} u d x \leqq G^{m}, v_{m} \leqq v \leqq v^{m}\right\},
$$

where $u_{0}, u^{0}, G_{0}, G^{0}, v_{0}$, and $v^{0}$ are

$$
\begin{gathered}
u_{0}=0, \quad G_{0}=v_{0}=1, \quad u^{0}=1, \quad G^{0}=1+\lambda V_{\Omega}+\mu^{-1} \sigma S_{\Omega}, \\
v^{0}=1+\mu^{-1} \phi^{2} V_{\Omega} \sup \{f(1, v)+h(1, v): v \geqq 1\},
\end{gathered}
$$


and where $u_{m}, u^{m}, G_{m}, G^{m}, v_{m}$, and $v^{m}(m \geqq 1)$ are defined inductively by

$$
\begin{aligned}
& \Delta u_{m}-\phi^{2} M u_{m}=\phi^{2}\left[f\left(u_{m-1}, v^{m-1}\right)-M u_{m-1}\right] \text { in } \Omega \text {, } \\
& \partial u_{m} / \partial n=\sigma\left(1-u_{m}\right) \text { on } \partial \Omega, \\
& \Delta u^{m}-\phi^{2} M u^{m}=\phi^{2}\left[f\left(u^{m-1}, v_{m-1}\right)-M u^{m-1}\right] \text { in } \Omega \text {, } \\
& \partial u^{m} / \partial n=\sigma\left(1-u^{m}\right) \quad \text { on } \partial \Omega \\
& G_{m}=1+\lambda \int_{\Omega} u_{m} d x+\mu^{-1} \sigma \int_{\partial \Omega}\left(1-u^{m}\right) d s, \\
& G^{m}=1+\int_{\Omega} u^{m} d x+\mu^{-1} \sigma \int_{\partial \Omega}\left(1-u_{m}\right) d s, \\
& v_{m}=\max \left\{1, G_{m}-\lambda \int_{\Omega} u^{m} d x\right. \\
& \left.1+\mu^{-1} \phi^{2} \int_{\Omega}\left[f\left(u_{m}, v_{m-1}\right)+h\left(u_{m}, v_{m-1}\right)-h\left(u^{m}, v^{m-1}\right)\right] d x\right\}, \\
& v^{m}=\min \left\{G^{m}-\lambda \int_{\Omega} u_{m} d x\right. \\
& \left.1+\mu^{-1} \phi^{2} \int_{\Omega}\left[f\left(u^{m}, v^{m-1}\right)+h\left(u^{m}, v^{m-1}\right)-h\left(u_{m}, v_{m-1}\right)\right] d x\right\}
\end{aligned}
$$

The sequence defined by (3.21)-(3.27) is such that (i) it approaches a solution of (3.13)-(3.18) as $m \rightarrow \infty$ (since it is seen to satisfy Lemma 3.2A and Theorem 3.3A), and (ii) it satisfies (3.19) for all $m \in N$ and for every solution of (3.13)-(3.18) (to prove it, use an induction argument, as above). Hence such sequence also approaches the maximal solution of (3.13)-(3.18) as $m \rightarrow \infty$, and the region $B$ of Theorem 3.3 may be obtained as the limit of the sequence of regions defined by (3.20), which may be easily computed (numerically in general) from the linear problems (3.23)-(3.27).

B. As it was mentioned in $\S 1$, since the region $B$ of Theorem 3.3 is globally asymptotically stable, it contains the nonwandering set of (1.1), (1.2), (2.1), and the same is true for any of the regions $B_{m}$ defined by (3.20)-(3.27) (since $B \subset B_{m}$ for all $m \in \mathbf{N}$, as it was seen in remark $A$ above). In particular every (stable or unstable) steady state of $(1.1),(1.2)$ is included in $B$.

C. If every solution of (3.13)-(3.18) satisfies $u_{*}=u^{*}$ and $v_{*}=v^{*}$, then the region $B$ of Theorem 3.3 is a singleton, $B=\left\{\left(u_{s}, v_{s}\right)\right\}$, and $\left(u_{s}, v_{s}\right)$ is a globally asymptotically stable steady state of $(1.1),(1.2)$; in addition, $\left(u_{s}, v_{s}\right)$ is the unique steady state of (1.1), (1.2), as it comes out from remark B above. Observe also that (3.13)-(3.18) has a unique solution in this case. This result will be used in the next section to obtain quantitative, sufficient conditions for global asymptotic stability and uniqueness of the steady state of (1.1), (1.2).

4. Global asymptotic stability of the steady state. In this section, we shall obtain sufficient conditions for global asymptotic stability of the steady state of (1.1), (1.2), (2.1), when the function $f$ is given by

$$
f(u, v)=g(u) \exp (\gamma-\gamma / v),
$$

where $g:\left[0, \infty\left[\rightarrow \mathbf{R}\right.\right.$ is a $C^{1}$-function satisfying

$$
g(0)=0, \quad g(u)>0 \quad \text { for all } u>0 .
$$


Particular instances of such form of $f$ are those in (1.4), (1.5). Some additional assumptions about the function $g$ will be considered below, when needed.

In order to avoid too many involved expressions, we shall obtain only reasonably good sufficient conditions for global stability (and not the best ones that can be obtained from the results of $\$ 3$ ).

The role of the parameters $\phi^{2}, \lambda$, and $\sigma$ deserves some attention. The Damköhler number $\phi^{2}$ is the basic parameter; the steady-state solutions of (1.1), (1.2), for example, are usually represented by the curve $\eta-\phi^{2}$, where $\eta$ is a significant functional of the steady state, i.e.,

$$
\eta=\int_{\Omega} f\left(u_{s}(x), v_{s}\right) d x / V_{\Omega} f(1,1),
$$

which is called the effectiveness factor (see [1]). Below, we shall prove that the steady state is globally asymptotically stable (i) if $\phi^{2}$ is sufficiently small or large, for fixed values of the remaining parameters; and (ii) for arbitrary values of $\phi^{2}$ if the parameter $\gamma$ is sufficiently small and the function $g$ is increasing. The parameter $\lambda$ is a Lewis number; increasing values of $\lambda$ are expected to make any steady state of (1.1), (1.2) more and more linearly unstable (i.e., to increase the growth rate of the linear stability analysis). This has been shown to be true for lumped chemically reacting systems (see [21]), and for some distributed systems (such as (1.1), (1.2) if $f(u, v)=u \exp (\gamma-\gamma / v)$; see [2]). Observe that the steady-state solutions of (1.1), (1.2) do not depend on $\lambda$. Some of the results below will be independent of $\lambda$ (they will be valid for $0<\lambda<\infty$ ), and some others (depending on $\lambda$ ) will be quite useful for small values of $\lambda$. The Sherwood number $\sigma$ is usually fairly large (see [1]). Some emphasis will be put on obtaining results that are significant as $\sigma \rightarrow \infty$ (see, e.g., Theorems 4.4 and 4.5 ).

Let us assume that the domain $\Omega$ satisfies assumption (H.1) (see Introduction). If the function $f$ is as defined by (4.1), then Theorem 3.3 applies. The system (3.13)(3.18) may be written as

$$
\begin{array}{r}
\Delta u_{*}=\phi^{2} g\left(u_{*}\right) \exp \left(\gamma-\gamma / v^{*}\right) \text { in } \Omega, \quad \partial u_{*} / \partial n=\sigma\left(1-u_{*}\right) \text { on } \partial \Omega, \\
\Delta u^{*}=\phi^{2} g\left(u^{*}\right) \exp \left(\gamma-\gamma / v_{*}\right) \text { in } \Omega, \quad \partial u^{*} / \partial n=\sigma\left(1-u^{*}\right) \text { on } \partial \Omega, \\
v_{*}=1+\max \left\{0,-\lambda \int_{\Omega}\left(u^{*}-u_{*}\right) d x+\mu^{-1} \sigma \int_{\partial \Omega}\left(1-u^{*}\right) d s,\right. \\
\mu^{-1} \phi^{2}\left[\exp \left(\gamma-\gamma / v_{*}\right) \int_{\Omega}\left(g\left(u_{*}\right)+h\left(u_{*}\right)\right) d x\right. \\
\left.\left.-\exp \left(\gamma-\gamma / v^{*}\right) \int_{\Omega} h\left(u^{*}\right) d x\right]\right\}, \\
v^{*}=1+\min \left\{\lambda \int_{\Omega}\left(u^{*}-u_{*}\right) d x+\mu^{-1} \sigma \int_{\partial \Omega}\left(1-u_{*}\right) d s,\right. \\
\mu^{-1} \phi^{2}\left[\exp \left(\gamma-\gamma / v^{*}\right) \int_{\Omega}\left(g\left(u^{*}\right)+h\left(u^{*}\right)\right) d x\right. \\
\left.\left.-\exp \left(\gamma-\gamma / v_{*}\right) \int_{\Omega} h\left(u_{*}\right) d x\right]\right\},
\end{array}
$$

where $h=[0,1] \rightarrow \mathbf{R}$ is a $C^{1}$-function satisfying

$$
h^{\prime}(u) \geqq 0, g^{\prime}(u)+h^{\prime}(u) \geqq 0 \quad \text { for all } 0 \leqq u \leqq 1 .
$$


The function $h$ may be chosen to be such that

$$
k_{1}=\max \left\{0, \max \left\{-g^{\prime}(u): 0 \leqq u \leqq 1\right\}\right\}=\max \left\{h^{\prime}(u): 0 \leqq u \leqq 1\right\} .
$$

The main idea to be used in the sequel is the following. According to Remark $3.4 \mathrm{C}$, if every solution of (4.3)-(4.7) satisfies

$$
u_{*}=u^{*}, \quad v_{*}=v^{*},
$$

then $(1.1),(1.2),(2.1)$ possess a unique steady state, which is globally asymptotically stable.

THEOREM 4.1 (Global asymptotic stability for small $\phi^{2}$ ). Under the assumptions above, (1.1), (1.2), (2.1) has a unique steady state, which is globally asymptotically stable if $\phi^{2}$ satisfies

$$
\phi^{2} \exp \gamma<\alpha / k_{3},
$$

and one of the following inequalities:

$$
\begin{aligned}
\gamma V_{\Omega}\left\{k_{2}\left(2 k_{1}+k_{5}\right)\left[k_{3}+k_{1} k_{4} \phi^{2} \exp \gamma /\left(\alpha-k_{1} \phi^{2} \exp \gamma\right)\right] \phi^{2}\right. \\
\left.\cdot \exp \gamma+k_{2}+2 k_{6}\right\} \phi^{2} \exp \gamma \leqq \mu,
\end{aligned}
$$

$$
\begin{aligned}
& \gamma k_{2}\left(2 \lambda \mu V_{\Omega}+\sigma S_{\Omega}\right)\left[k_{3}+k_{1} k_{4} \phi^{2} \exp \gamma /\left(\alpha-k_{1} \phi^{2} \exp \gamma\right)\right] \phi^{2} \exp \gamma \leqq \mu, \\
& \gamma k_{2} V_{\Omega}\left\{1+\left(2 \lambda \mu+k_{5} \phi^{2} \exp \gamma\right)\left[k_{3}+k_{1} k_{4} \phi^{2} \exp \gamma /\left(\alpha-k_{1} \phi^{2} \exp \gamma\right)\right] \phi^{2}\right.
\end{aligned}
$$

where $\alpha, k_{1}, k_{2}, k_{3}$, and $k_{4}$ are as in Lemma A.1 (see Appendix), and

$$
k_{5}=\max \left\{g^{\prime}(u): 0 \leqq u \leqq 1\right\}, \quad k_{6}=\max \{h(u): 0 \leqq u \leqq 1\} .
$$

Proof. We shall prove that if (4.11) and one of the inequalities (4.12)-(4.14) hold, then every solution of (4.3)-(4.7) satisfies (4.10). To this end, observe that if (4.11) holds, then $u_{*}$ and $u^{*}$ satisfy (Lemma A.1)

$$
u^{*}-u_{*} \leqq k_{2}\left[k_{3}+k_{1} k_{4} \phi^{2} \exp \gamma /\left(\alpha-k_{1} \phi^{2} \exp \gamma\right)\right] \phi^{2} \exp \gamma[1-\exp (-\gamma \xi)],
$$
where

$$
\xi=1 / v_{*}-1 / v^{*}
$$

is such that

$$
0 \leqq \xi \leqq 1, \quad \xi /(1-\xi) \leqq v_{*}^{2} \xi /\left(1-v_{*} \xi\right)=v^{*}-v_{*},
$$

as it comes out from (4.7). Subtraction of (4.5) from (4.6) yields

$$
\begin{aligned}
v^{*}-v_{*} \leqq \mu^{-1} \phi^{2}\left[\exp \left(\gamma-\gamma / v^{*}\right) \int_{\Omega}\left(g\left(u^{*}\right)+2 h\left(u^{*}\right)\right) d x\right. \\
\left.-\exp \left(\gamma-\gamma / v_{*}\right) \int_{\Omega}\left(g\left(u_{*}\right)+2 h\left(u_{*}\right)\right) d x\right],
\end{aligned}
$$

$$
v^{*}-v_{*} \leqq 2 \lambda \int_{\Omega}\left(u^{*}-u_{*}\right) d x+\mu^{-1} \sigma \int_{\partial \Omega}\left(u^{*}-u_{*}\right) d s .
$$

Integration over $\Omega$ in (4.3) and (4.4) and application of Green's identity yield

$$
\begin{aligned}
& \sigma \int_{\partial \Omega}\left(1-u_{*}\right) d s=\phi^{2} \exp \left(\gamma-\gamma / v^{*}\right) \int_{\Omega} g\left(u_{*}\right) d x, \\
& \sigma \int_{\partial \Omega}\left(1-u^{*}\right) d s=\phi^{2} \exp \left(\gamma-\gamma / v_{*}\right) \int_{\Omega} g\left(u^{*}\right) d x .
\end{aligned}
$$


Substraction of (4.21) from (4.20) and substitution in (4.19) lead to

$$
\begin{aligned}
& v^{*}-v_{*} \leqq 2 \lambda \int_{\Omega}\left(u^{*}-u_{*}\right)+\mu^{-1} \phi^{2}\left[\exp \left(\gamma-\gamma / v^{*}\right) \int_{\Omega} g\left(u_{*}\right) d x\right. \\
&\left.-\exp \left(\gamma-\gamma / v_{*}\right) \int_{\Omega} g\left(u^{*}\right) d x\right] .
\end{aligned}
$$

Finally, after substitution of (4.15)-(4.17) in (4.18), (4.19), and (4.22), the following inequalities are obtained:

$$
\xi /(1-\xi) \leqq A_{i}[1-\exp (-\gamma \xi)] \text { for } i=1,2 \text {, and } 3 \text {, }
$$

where $\gamma \mu A_{1}, \gamma \mu A_{2}$, and $\gamma \mu A_{3}$ are the first members of (4.12), (4.13), and (4.14). If one of the inequalities (4.12) $-(4.14)$ is satisfied, then $\xi=0$ (i.e., $v_{*}=v^{*}$ ), as it comes out from (4.23), $u_{*}=u^{*}$ (Lemma A.1) and the conclusion of the theorems follows.

Remark. Condition (4.12) does not depend on $\lambda$, and it is more stringent than (4.14) if $k_{\mathrm{L}} \neq 0$ and $\lambda$ is sufficiently small. If $\sigma$ is sufficiently small, condition (4.14) is more stringent than (4.13).

THEOREM 4.2 (Global asymptotic stability for all $\phi^{2}>0$ ). If, in addition to the assumptions of Theorem 4.1, the function g satisfies condition (A.6) of Lemma A.2 (see Appendix), then, for all $\phi^{2}>0,(1.1),(1.2),(2.1)$ have a unique steady state, which is globally asymptotically stable, provided that $\gamma$ satisfies one of the following inequalities (see Fig. 1):

$$
\begin{gathered}
k_{7} \gamma \sigma S_{\Omega} / \mu \leqq 1 /\left(1+2 \lambda \mu V_{\Omega} / \sigma S_{\Omega}\right) \\
\gamma \sigma S_{\Omega} / \mu \leqq 1 /\left(1+k_{7} \lambda \mu V_{\Omega} / \sigma S_{\Omega}\right)
\end{gathered}
$$
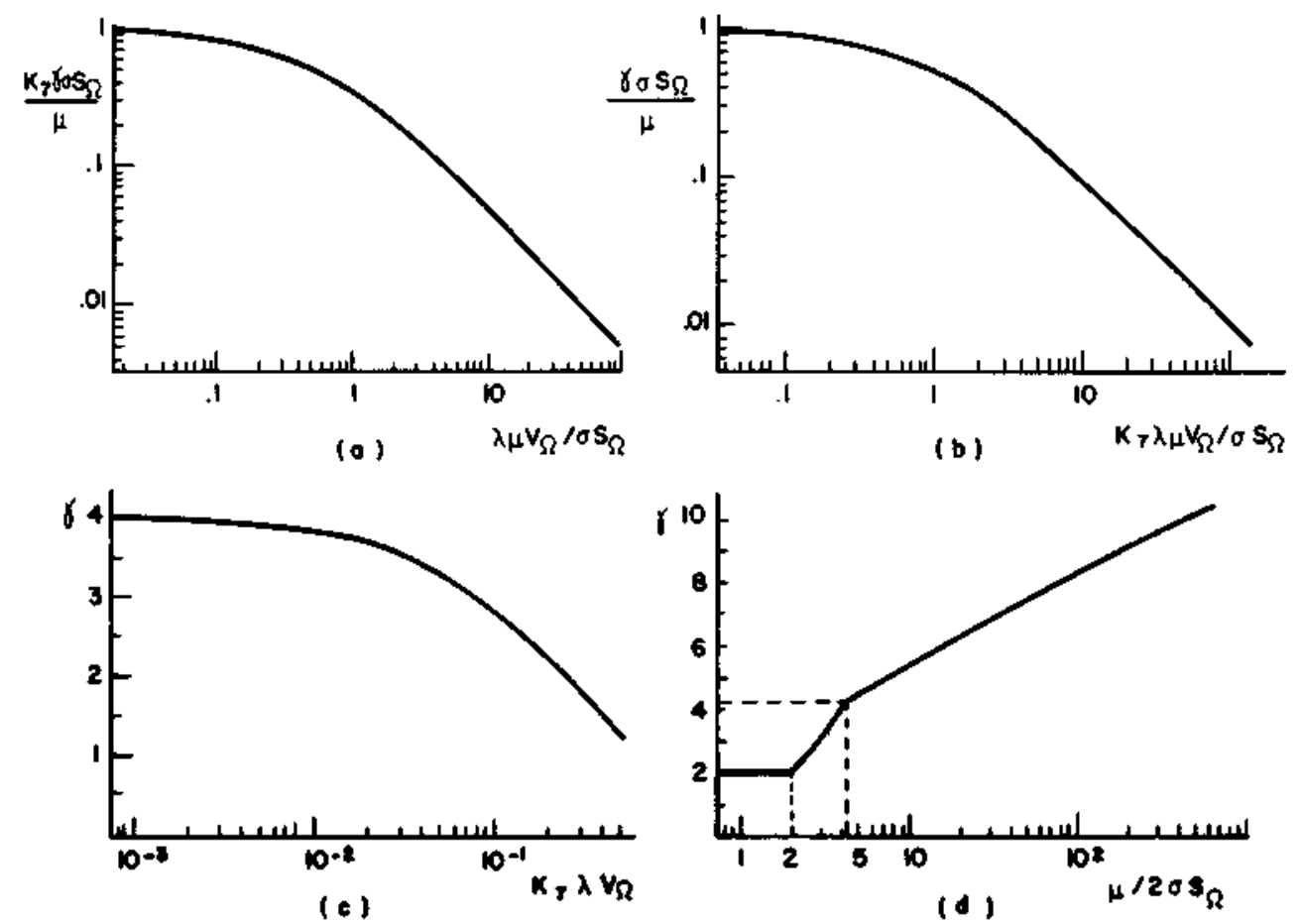

FIo. I. Global asymptotic stability for all $\phi^{2}>0$. Plots (a)-(d) correspond to conditions $(4.24)-(4.27)$. 


$$
\begin{gathered}
\gamma \leqq 4 /\left(1+4 k_{7} \lambda V_{\Omega}\right), \\
\gamma \leqq H_{1}\left(\mu / 2 \sigma S_{\Omega}\right) .
\end{gathered}
$$

Here, $k_{7}$ is as defined in Lemma A.2, and the positive, nondecreasing function $H_{1}:[0, \infty[\rightarrow$ $\mathbf{R}$ is defined by $H_{1}(y)=2$ for $0 \leqq y \leqq 2, H_{1}(y)=y$ for $2<y \leqq y_{1}$, and $H_{1}(y)=h_{2}\left[h_{1}^{-1}(y)\right]$ for $y_{t}<y<\infty$, where (i) $y_{1}=h_{1}\left(z_{t}\right)=4.2488 \cdots$ and $z_{1}=2.6761 \cdots$ is the unique positive solution of the equation $z^{2}=\sinh z$ tanh $z$, and (ii) the strictly increasing functions, $h_{1}$, $h_{2}:\left[z_{1}, \infty\left[\rightarrow\left[y_{1}, \infty[\right.\right.\right.$ are given by

$$
h_{1}(z)=\sinh z \tanh z /(z-\tanh z), \quad h_{2}(z)=z^{2} /(z-\tanh z) .
$$

Proof. If Lemma A.2 is applied to (4.3), (4.4), one obtains

$$
0 \leqq u^{*}-u_{*}<1-\exp \left(-k_{r} \gamma \xi\right)
$$

where $\xi$ is given by (4.16) and satisfies (4.17) and

$$
\begin{gathered}
1 / v_{*} v^{*} \leqq 1-\xi, \quad\left(v_{*}-1\right) / v_{*} v^{*}<(1-\xi)^{2} / 4, \\
\left(v^{*}-1\right) /\left(v_{*}-1\right) \geqq(1+\xi)^{2} /(1-\xi)^{2},
\end{gathered}
$$

as is easily seen when taking into account (4.7).

When using (4.20), (4.21), the following inequalities are obtained from (4.5), (4.6), upon subtraction or division,

$$
\begin{gathered}
v^{*}-v_{*} \leqq 2 \lambda \int_{\Omega}\left(u^{*}-u_{*}\right) d x+\mu^{-1} \sigma \int_{\partial \Omega}\left(u^{*}-u_{*}\right) d s \\
v^{*}-v_{*} \leqq \lambda \int_{\Omega}\left(u^{*}-u_{*}\right) d x+\mu^{-1} \sigma[1-\exp (-\gamma \xi)] \int_{\partial \Omega}\left(1-u_{*}\right) d s \\
v^{*}-v_{*} \leqq(\sigma / \mu)\left[\exp (\gamma \xi) \int_{\partial \Omega}\left(1-u^{*}\right) d s-\exp (-\gamma \xi) \int_{\partial \Omega}\left(1-u_{*}\right) d s\right] \\
\left(v^{*}-1\right) /\left(v_{*}-1\right) \leqq \exp (2 \gamma \xi)\left[\int_{\partial \Omega}\left(1-u^{*}\right) d s\right] /\left[\int_{\partial \Omega}\left(1-u_{*}\right) d s\right]
\end{gathered}
$$

(Recall that the function $h$ identically vanishes since $g^{\prime}(u)>0$ for all $0<u \leqq 1$, according to condition (A.6) of Lemma A.2.) A further substitution of (4.5), (4.20) into (4.31) yields

$$
v^{*}-v_{*} \leqq \lambda \int_{\Omega}\left(u^{*}-u_{*}\right) d x+\left(v_{*}-1\right)[\exp (\gamma \xi)-1]
$$

When taking into account (4.7), (4.16), (4.28), (4.29), the following inequalities are obtained from $(4.30)-(4.34)$ :

$$
\begin{aligned}
& \xi /(1-\xi) \leqq\left(2 \lambda V_{\Omega}+\sigma S_{\Omega} / \mu\right)\left[1-\exp \left(-k_{7} \gamma \xi\right)\right], \\
& \xi /(1-\xi) \leqq \lambda V_{\Omega}\left[1-\exp \left(-k_{7} \gamma \xi\right)\right]+\left(\sigma S_{\Omega} / \mu\right)[1-\exp (-\gamma \xi)], \\
& \xi /(1-\xi)<\left(2 \sigma S_{\Omega} / \mu\right) \sinh (\gamma \xi), \quad(1+\xi) /(1-\xi)<\exp (\gamma \xi) \quad \text { if } \xi>0, \\
& \xi /(1-\xi) \leqq \lambda V_{\Omega}\left[1-\exp \left(-\gamma k_{7} \xi\right)\right]+(1-\xi)[\exp (\gamma \xi)-1] / 4 .
\end{aligned}
$$

If inequality (4.24) $((4.25)$ or (4.26), respectively) holds, then (4.35) ((4.36) or (4.38), respectively) yields $\xi=0$ (i.e., $v_{*}=v^{*}$ ); then $u_{*}=u^{*}$ (apply Lemma A.1 and take into account that $k_{1}=0$ ) and the conclusion of the theorem follows. If (4.27) holds, then $\xi=0$ and the conclusion of the theorem follows again. Use the second inequality (4.37) 
if $\gamma \leqq 2$ to prove it, and observe that if $\gamma>2$ and $\xi>0$, then (4.27) and the first inequality (4.37) yield

$$
H_{1}^{-1}(\gamma)<(1 / \xi-1) \sinh (\gamma \xi) ;
$$

but this inequality cannot be satisfied for any $\xi>0$ since the maximum of its second member, in $0 \leqq \xi \leqq 1$, is $H_{1}^{-1}(\gamma)$.

THEOREM 4.3 (Global asymptotic stability for large $\phi^{2}$ ). In addition to the assumptions of Theorem 4.1, let the function g satisfy conditions (A.7) and (A.8) of Lemma A.3. Then, (1.1), (1.2), (2.1) have a unique steady state, which is globally asymptotically stable for

$$
\phi^{2} \geqq \phi_{c}^{2}=\sigma\left(\sigma+p / \rho_{1}\right) / G(\delta),
$$

if $\delta$ is such that $0<\delta \leqq a$ and satisfies one of the following inequalities:

$$
\begin{gathered}
\gamma \leqq \gamma_{c}=(1-\delta) H_{2}\left(a_{1}(1-\delta) / 2+1 / 2 a_{1}(1-\delta)\right), \\
\delta \leqq \max \left\{\left(1+1 / a_{1}\right) /\left[1+\gamma k_{8}\left(1+2 a_{2}\right)\right], 2 a_{3} /\left[a_{4}+\sqrt{a_{4}^{2}-4 a_{3}}\right]\right\},
\end{gathered}
$$

where (i) the strictly increasing function $G$ and the constants $\rho_{1}, a$, and $k_{8}$ are as in Lemma A.3; (ii) the strictly increasing function $\mathrm{H}_{2}:[1, \infty[\rightarrow[2, \infty[$ (see Fig. 2) is given by $H_{2}(y)=1+y$ for $1 \leqq y \leqq 2, H_{2}(y)=h_{3}\left[h_{4}^{-1}(y)\right]$ for $2<y<\infty$; (iii) the strictly increasing functions $h_{3}:\left[0, \infty\left[\rightarrow\left[3, \infty\left[\right.\right.\right.\right.$ and $h_{4}:[0, \infty[\rightarrow[2, \infty[$ are defined by

$h_{3}(z)=z^{2} \sinh z /(z \cosh z-\sinh z), \quad h_{4}(z)=(\sinh z \cosh z-z) /(z \cosh z-\sinh z)$; and (iv) the parameters $a_{1}, a_{2}, a_{3}$, and $a_{4}$ are

$$
\begin{gathered}
a_{1}=\sigma S_{\Omega} / \mu, \quad a_{2}=\lambda \mu V_{\Omega} / \sigma S_{\Omega}, \quad a_{3}=\left(1+1 / a_{1}^{2}\right) /\left(1+a_{2}\right), \\
a_{4}=\left[\left(1+a_{1}\right)\left(2+a_{2}\right)+\gamma k_{8}\left(1+2 a_{2}\right)\right] / a_{1}\left(1+a_{2}\right) .
\end{gathered}
$$

Proof. If (4.39) holds and $0<\delta \leqq a$, then (Lemma A.3)

$$
0 \ll u_{*} \leqq u^{*}<\delta \leqq a, \quad 0 \leqq u^{*}-u_{*} \leqq \delta\left[1-\exp \left(-\gamma k_{8} \xi\right)\right],
$$

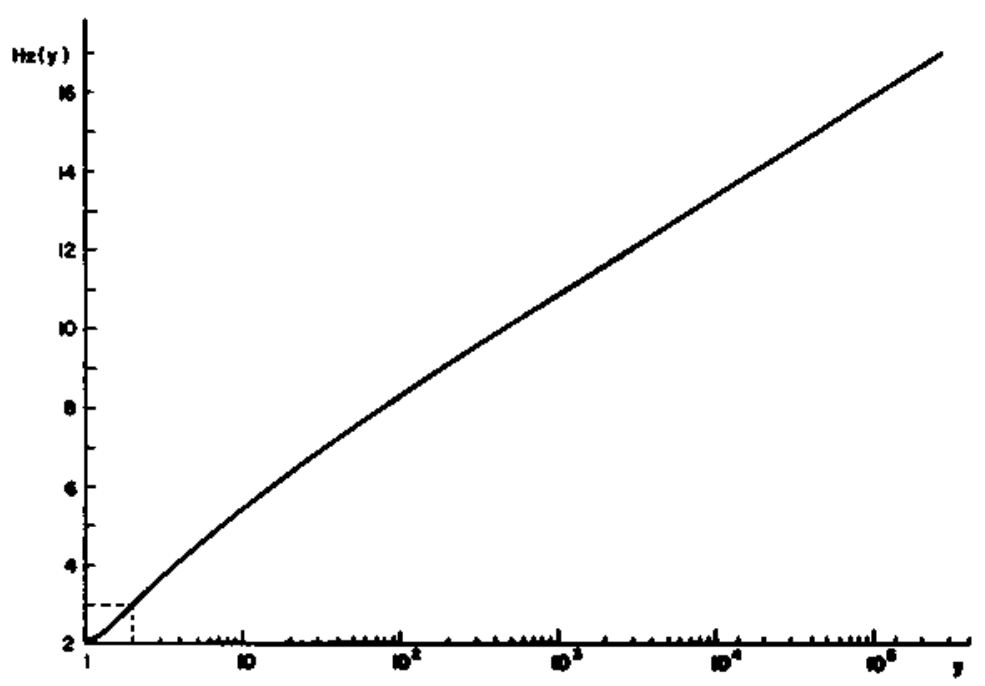

FIG. 2. The function $\mathrm{H}_{2}$ of Theorems 4.3 and 4.4 . 
where $\xi=1 / v_{*}-1 / v^{*} \leqq 1$. Then if the function $h$ is chosen to be such that $h(u)=0$ for $0 \leqq u \leqq a$ (this may be done, with $h$ satisfying (4.8), (4.9), since the function $g$ satisfies (A.7)),

$$
h\left(u_{*}(x)\right)=h\left(u^{*}(x)\right)=0 \text { for all } x \in \bar{\Omega} .
$$

Let us first assume that $\phi^{2}$ and $\delta$ satisfy (4.39), (4.40) and prove that $\xi=0$. To this end, we define

$$
\begin{aligned}
& A_{*}=1+\mu^{-1} \sigma \exp (-\gamma \xi) \int_{\partial \Omega}\left(1-u_{*}\right) d s, \\
& A^{*}=1+\mu^{-1} \sigma \exp (\gamma \xi) \int_{\partial \Omega}\left(1-u^{*}\right) d s .
\end{aligned}
$$

$A_{*} \leqq v_{*}$ and $A^{*} \geqq v^{*}$, as it comes out from (4.5), (4.6), (4.20), (4.21), (4.43). Hence, if $\xi$ were different from zero, it would satisfy

$$
\begin{aligned}
\xi & =1 / v_{*}-1 / v^{*} \leqq\left(A^{*}-A_{*}\right) / A_{*} A^{*} \\
& <2 a_{1} \sinh (\gamma \xi) /\left[1+a_{1}(1-\delta) \exp (-\gamma \xi)\right]\left[1+a_{1}(1-\delta) \exp (\gamma \xi)\right],
\end{aligned}
$$

or

$$
1>(1-\delta) \xi\left[a_{1}(1-\delta) / 2+1 / 2 a_{1}(1-\delta)+\cosh \gamma \xi\right] / \sinh \gamma \xi,
$$

as obtained from (4.42). (4.44). But this inequality cannot hold for any $\xi>0$ since the minimum of its second member, in $0 \leq \xi<\infty$, is $(1-\delta) H_{2}\left(a_{1}(1-\delta) / 2+1 / 2 a_{1}(1-\delta)\right) / \gamma$, and $\gamma$ satisfies (4.40). Then, $\xi=0$ (i.e., $\left.v_{*}=v^{*}\right), u_{*}=u^{*}$ (Lemma A.3) and the conclusion of the theorem follows.

If $\phi^{2}$ and $\delta$ satisfy (4.39) and (4.41), then

$$
v_{*} \geqq \max \left\{1,1+a_{1}\left(1-\delta-a_{2} \delta\right)\right\}, \quad v^{*}>1+a_{1}(1-\delta),
$$

as it comes out from $(4.5),(4.6),(4.21),(4.42)$. If $\xi$ were different from zero, $(4.30)$, (4.42), (4.46) would yield

$$
1<a_{1}\left(1+2 a_{2}\right) \delta\left[1-\exp \left(-k_{8} \gamma \xi\right)\right] / \xi\left[1+a_{1}(1-\delta)\right] \max \left\{1,1+a_{1}\left(1-\delta-a_{2} \delta\right)\right\} .
$$

But this inequality cannot hold for any $\xi>0$ if $\delta$ satisfies (4.41), as is easily seen. Therefore, $\xi=0$ and the conclusion of the theorem follows again.

Remarks. If $\phi_{c}^{2}$ is calculated by means of (4.39), (4.40), then it does not depend on $\lambda$, while if it is obtained from (4.39), (4.41), then $\phi_{c}^{2} \rightarrow \infty$ as $\lambda \rightarrow \infty$.

It is easily seen that, for fixed values of the remaining parameters, the functions $\delta \rightarrow \phi_{c}^{2}(\delta)$ and $\delta \rightarrow \gamma_{c}(\delta)$ are strictly decreasing in $0<\delta<1$. Therefore, if

$$
\gamma<\mathrm{H}_{2}\left(a_{1} / 2+1 / 2 a_{1}\right),
$$

then the maximum value of $\delta$ satisfying $(4.40), \delta_{M}$, is the unique solution of the equation $\gamma=\gamma_{c}(\delta)$. Then, the best value of $\phi_{c}^{2}$ provided by $(4.39),(4.40)$ is $\sigma(\sigma+$ $\left.p / \rho_{1}\right) / G(\delta)$, with $\delta=\min \left\{a, \delta_{M}\right\}$. If $(4.47)$ does not hold, then (4.40) is not satisfied for any $\delta>0$, and Theorem 4.3 does not provide a value of $\phi_{c}^{2}$ uniformly valid in $0<\lambda<\infty$. Although Theorem 4.3 provides only sufficient conditions for global asymptotic stability of the steady state, it may be seen, as a converse of Theorem 4.3 in a certain sense, that for first-order Arrhenius kinetics (i.e., for $g(u) \equiv u$ ) and large values of $\sigma$ (see [2]), the upper linear instability bound (i.e., the supremum of the set of values of $\phi^{2}$ such that the steady state of $(1.1),(1.2),(2.1)$ is linearly unstable), $\phi_{u}^{2}$, satisfies $\phi_{u}^{2} \rightarrow \phi_{u 0}^{2}<\infty$ if $\gamma<\left(1+a_{1}\right)^{2} / a_{1}$ and $\phi_{u}^{2} \rightarrow \infty$ otherwise as $\lambda \rightarrow \infty$. 
Any $\phi_{c}^{2}$ provided by (4.39), (4.40), or by (4.39), (4.41), is such that $\phi_{c}^{2} \rightarrow \infty$ as $\sigma \rightarrow \infty$. In order to calculate a value of $\phi_{c}^{2}$ uniformly valid in $0<\sigma<\infty$, which is expected to exist under mild assumptions on the function $\mathrm{g}$, one would need the following result, which is stronger than that in Lemma A.3 and seemingly true (under mild assumptions on the function $g$ ): there are two constants, $\bar{\Lambda}$ and $k$, such that, for every $\sigma>0$, (i) the problem (A.1) of the Appendix has a unique solution if $\Lambda \geqq \bar{\Lambda}$, and (ii) if $\bar{\Lambda} \leqq \Lambda_{2}<\Lambda_{1}<\infty$, then the solutions of (A.1) for $\Lambda=\Lambda_{1}$ and $\Lambda=\Lambda_{2}, u_{1}$ and $u_{2}$, satisfy $\left|u_{2}(x)-u_{1}(x)\right| \leqq k\left(\Lambda_{1}-\Lambda_{2}\right)$, for all $x \in \bar{\Omega}$. Property (i) may be proved if one is able to obtain an upper multiplicity bound $\bar{\Lambda}$ when the Robin boundary data in (A.1) is replaced by Dirichlet data: $u=1$ on $\partial \Omega$; if $\Omega$ is the unit ball of $\mathbf{R}^{p}$, this comes out from results by Dancer [23] that were obtained by means of topological degree theory; unfortunately, even if the results of [23] are extended to arbitrary bounded domains of $\mathbf{R}^{p}$, they do not seem to provide the constant $k$ of part (ii) of the required result above. Related results in the literature, such as those in [24], [25], do not apply to our case.

Theorems 4.4 and 4.5 below provide a uniform value of $\phi_{c}^{2}$ in $0<\sigma<\infty$ but they require the function $g$ to be strictly increasing.

THEOREM 4.4 (Global asymptotic stability for large $\phi^{2}$ ). In addition to the assumptions of Theorem 4.1, let us assume that $g$ is such that $g^{\prime}(u)>0$ for all $0<u \leqq 1$, and that

$$
2<\gamma \leqq H_{2}\left(a_{1} / 2+1 / 2 a_{1}\right),
$$

where the constant $a_{1}$ and the function $H_{2}$ (see Fig. 2) are as defined in Theorem 4.3. Then, (1.1), (1.2), (2.1) have a unique steady state, which is globally asymptotically stable if

$$
\phi^{2} \geqq 2\left(\mu / S_{\Omega}\right)^{2} K\left[p S_{\Omega} / \mu \rho_{1}+2^{-1 / p} K\right] / G\left(\delta_{1}\right),
$$

where the constants $\delta, \delta_{1}$, and $K$ are the unique solutions of the equations

$$
\begin{aligned}
& \gamma=(1-\delta) H_{2}\left[a_{1}(1-\delta) / 2+1 / 2 a_{1}(1-\delta)\right], \quad 0<\delta<1, \\
& \left(1-\delta_{1}\right) / \sqrt{2 G\left(\delta_{1}\right)}=\left(1+D / 2 \rho_{2}\right)^{p-1} \sqrt{\left(1+p / \sigma \rho_{1}\right) / G(\delta)}, \quad 0<\delta_{1}<1, \\
& H_{2}(K / 2+2 / K)=\gamma a_{5}, \quad K \geqq 1,
\end{aligned}
$$

the constants $\rho_{1}, \rho_{2}$, and $D$ are as defined in Lemma A.4, and

$$
\begin{aligned}
& a_{5}=\left(1+D / 2 \rho_{2}\right)^{p-1}\left[p / \rho_{1}+\sqrt{\left(p / \rho_{1}\right)^{2}+2^{1-1 / p} a_{6}}\right] \sqrt{2 G(1) / a_{6} G\left(\delta_{1}\right)}, \\
& a_{6}=2\left(\mu / S_{\Omega}\right)^{2}\left[p S_{\Omega} / \mu \rho_{1}+2^{-1 / p}\right] .
\end{aligned}
$$

Remarks. If $\gamma \leqq 2$, then the conclusion of the thearem is true for all $\phi^{2}>0$, according to Theorem 4.2. Equation (4.50) has a unique solution if $\gamma$ satisfies (4.48), as was seen in a remark above. For a given value of $\delta,(4.51)$ has a unique solution $\delta_{1}$ (which is such that $\delta_{1}<\delta$ ), since the first member of (4.51) is a strictly decreasing function of $\delta_{1}$, and it approaches 0 and $\infty$ as $\delta_{1} \rightarrow 1$ and as $\delta_{1} \rightarrow 0$, respectively. Since the second member of (4.52) is larger than $2\left(a_{5}>1\right.$ and $\left.\gamma>2\right),(4.52)$ has a unique solution (recall that $H_{2}(1)=2, H_{2}$ is strictly increasing and $H_{2}(y) \rightarrow \infty$ as $y \rightarrow \infty$ ).

Proof of Theorem 4.4. If $\phi^{2} G(\delta) \exp \left(\gamma-\gamma / v_{*}\right) \geqq \sigma\left(\sigma+p / \rho_{1}\right)$, then $u_{*} \leqq u^{*} \leqq \delta$ (Lemma A.3) and, as in the proof of Theorem 4.3, $\xi=1 / v_{*}-1 / v^{*}$ is seen to satisfy (4.45), which implies $\xi=0$ (i.e., $v_{*}=v^{*}$ ). Then $u_{*}=u^{*}$ (Lemma A.1), and the conclusion of the theorem follows.

If $\phi^{2} G(\delta) \exp \left(\gamma-\gamma / v_{*}\right)<\sigma\left(\sigma+p / \rho_{1}\right)$, then $u_{m}^{*}=\min \left\{u^{*}(x): x \in \partial \Omega\right\}$ satisfy (Lemma A.4)

$$
\left(1-u_{m}^{*}\right) / \sqrt{2 G\left(u_{m}^{*}\right)}<\left(1+D / 2 \rho_{2}\right)^{p-1} \sqrt{\left(1+p / \sigma \rho_{1}\right) / G(\delta)} .
$$


Since the first member of (4.53) is a strictly decreasing function of $u_{m}^{*}$ and $\delta_{1}$ satisfies (4.51), $\delta_{1} \leqq u_{m}^{*}$ and

$$
\delta_{1}<u^{*}(x) \text { for all } x \in \partial \Omega
$$

Then, $u_{m}^{*}$ and $u_{M}^{*}=\max \left\{u^{*}(x): x \in \partial \Omega\right\}=\max \left\{u^{*}(x): x \in \bar{\Omega}\right\}$ satisfy (Lemma A.4)

$$
\begin{gathered}
\sigma\left(1-u_{m}^{*}\right)<\left(1+D / 2 \rho_{2}\right)^{p-1} \sqrt{2 \Lambda_{*} G(1)} \\
\sigma\left(1-u_{M}^{*}\right)>\Lambda_{*} G\left(\delta_{1}\right) /\left[p / \rho_{1}+\sqrt{\left(p / \rho_{1}\right)^{2}+2^{1-1 / p} \Lambda_{*} G\left(\delta_{1}\right)}\right]=\mu K_{*} / S_{\Omega}
\end{gathered}
$$

where

$$
\Lambda_{*}=\phi^{2} \exp \left(\gamma-\gamma / v_{*}\right)
$$

Since $K \geqq 1$ and $\phi^{2}$ satisfies (4.49), we have $\Lambda_{*} G\left(\delta_{1}\right) \geqq \phi^{2} G\left(\delta_{1}\right) \geqq a_{6}$, and

$$
\left(1+D / 2 \rho_{2}\right)^{p-1} \sqrt{2 \Lambda_{*} G(1)} S_{\Omega} / \mu K_{*} \leqq a_{5} .
$$

Furthermore, (4.49), (4.56), (4.57) yield

$$
K \leqq K_{*} \text {. }
$$

Then, if $A_{*}$ and $A^{*}$ are as defined by (4.44), $\xi=1 / v_{*}-1 / v^{*} \leqq\left(A^{*}-A_{*}\right) / A_{*} A^{*}$ must vanish because otherwise it would satisfy

$$
\xi<a_{5} \sinh (\gamma \xi) /(K / 2+1 / 2 K+\cosh \gamma \xi),
$$

as it comes out from (4.44), (4.55), (4.56), (4.58), (4.59), or

$$
a_{s}>(K / 2+1 / 2 K+\cosh \gamma \xi) / \sinh \gamma \xi,
$$

and this inequality cannot hold for any $\xi>0$ since the minimum of its second member in $0 \leqq \xi<\infty$ is $H_{2}(K / 2+1 / 2 K) / \gamma$, and $K$ satisfies (4.52). Therefore, $\xi=0$ (i.e., $v_{*}=v^{*}$ ), $u_{*}=u^{*}$ (Lemma A.1) and the conclusion of the theorem follows.

Observe that the second member of (4.49) does not depend on $\lambda$. The following theorem provides a better result if $\lambda$ is sufficiently small. It also applies for arbitrarily large values of $\sigma$.

THEOREM 4.5 (Global asymptotic stability for large $\phi^{2}$ ). In addition to the hypothesis of Theorem 4.1, let us assume that the function $g$ satisfies condition (A.6) of Lemma A.2, and that

(4.60) $\gamma>2, \quad \delta=\max \left\{\left(1+1 / a_{1}\right) /\left[1+\gamma k_{7}\left(1+2 a_{2}\right)\right], 2 a_{3} /\left[a_{7}+\sqrt{a_{7}^{2}-4 a_{3}}\right]\right\}<1$, where the constants $a_{1}, a_{2}$, and $a_{3}$ are as in Theorem 4.3, $k_{7}$ is as defined in Lemma A.2, and

$$
a_{7}=\left[\left(1+a_{1}\right)\left(2+a_{2}\right)+\gamma k_{7}\left(1+2 a_{2}\right)\right] / a_{1}\left(1+a_{2}\right) .
$$

Then, (1.1), (1.2), (2.1) possess a unique solution, which is globally asymptotically stable if

$$
\phi^{2} \geqq 2\left(\mu / S_{\Omega}\right)^{2} K\left[p S_{\Omega} / \mu \rho_{1}+2^{-1 / \rho} K\right] / G\left(\delta_{1}\right),
$$

where $\delta_{\mathfrak{l}}$ is the unique solution of

$$
\left(1-\delta_{1}\right) / \sqrt{2 G\left(\delta_{1}\right)}=\left(1+D / 2 \rho_{2}\right)^{p-1} \sqrt{\left(1+p / \sigma \rho_{1}\right) / G(\delta)}, \quad 0<\delta_{1}<1,
$$

the constants $\rho_{1}, \rho_{2}$, and $D$ are as in Lemma A.4, and

$$
K=\left[\gamma-2+\lambda V_{\Omega}+\sqrt{\left(\gamma+\lambda V_{\Omega}\right)^{2}+4 \gamma \lambda k_{7} V_{\Omega}}\right] / 2 \text {. }
$$

Remark. If $\gamma \leqq 2$ or if $\delta \geqq 1$, then the conclusion of the theorem is true for all $\phi^{2}>0$, according to Theorem 4.2 . 
Proof. If $\phi^{2} G(\delta) \exp \left(\gamma-\gamma / v_{*}\right) \geqq \sigma\left(\sigma+p / \rho_{1}\right)$, then $u_{*} \leqq u^{*} \leqq \delta$ (Lemma A.3) and, as in the proof of Theorem $4.3, \xi=1 / v_{*}-1 / v^{*}$ is seen to satisfy

$$
\xi \leqq a_{1}\left(1+2 a_{2}\right) \delta\left[1-\exp \left(-k_{7} \gamma \xi\right)\right] /\left[1+a_{1}(1-\delta)\right] \max \left\{1,1+a_{1}\left(1-\delta-a_{2} \delta\right)\right\} \text {. }
$$

This inequality cannot hold for any $\xi>0$ if $\delta$ is given by (4.60). Therefore, $\xi=0$ (i.e., $\left.v_{*}=v^{*}\right), u_{*}=u^{*}$ (Lemma A.1) and the conclusion of the theorem follows.

If $\phi^{2} G(\delta) \exp \left(\gamma-\gamma / v_{*}\right)<\sigma\left(\sigma+p / \rho_{1}\right)$, then $u_{m}^{*}=\min \left\{u^{*}(x): x \in \partial \Omega\right\}$ satisfies (4.53) (Lemma A.4). As in the proof of Theorem 4.4, this implies that $u^{*}$ satisfies (4.54). Then $u_{m}^{*}$ and $u_{M}^{*}=\max \left\{u^{*}(x): x \in \partial \Omega\right\}$ are seen to satisfy (4.55), (4.56), where $\Lambda_{*}$ is given again by (4.57), and $K$ satisfies (4.59) again. In addition, $u_{*}$ and $u^{*}$ satisfy (4.28) (Lemma A.2). Then $B_{*}$ and $B^{*}$, which are defined by

$$
\begin{gathered}
B_{*}=1-\lambda \int_{\Omega}\left(u^{*}-u_{*}\right) d x+\mu^{-1} \sigma \int_{\partial \Omega}\left(1-u^{*}\right) d s, \\
B^{*}=1+\mu^{-1} \sigma \exp (\gamma \xi) \int_{\partial \Omega}\left(1-u^{*}\right) d s,
\end{gathered}
$$

satisfy

$$
\begin{gathered}
0 \leqq B^{*}-B_{*} \leq \lambda \int_{\Omega}\left(u^{*}-u_{*}\right) d x+\left(B^{*}-1\right)[1-\exp (-\gamma \xi)], \\
B_{*} \geqq 1+K-\lambda V_{\Omega}, \quad B^{*} \geqq 1+K
\end{gathered}
$$

as it comes out from (4.56), (4.59). Also, $B_{*} \leqq v_{*}$ and $B^{*} \geqq v^{*}$ (see (4.5), (4.6), (4.21)). Hence, $\xi=1 / v_{*}-1 / v^{*} \leqq\left(B^{*}-B_{*}\right) / B_{*} B^{*}$ satisfies

$$
\xi \leqq[1-\exp (-\gamma \xi)] /\left(1+K-\lambda V_{\Omega}\right)+\lambda V_{\Omega}\left[1-\exp \left(-k_{7} \gamma \xi\right)\right] /(1+K)\left(1+K-\lambda V_{\Omega}\right),
$$

as obtained from (4.28), (4.62). But this inequality cannot hold for any $\xi>0$ if $K$ is given by (4.61), as it is easily seen. Therefore $\xi=0$ and the conclusion of the theorem follows.

Finally, since the steady-state solutions of (1.1), (1.2) do not depend on the parameter $\lambda$, the following corollary is true.

COROLLARY 4.6. If, for some $\lambda>0$, the hypothesis of one of the Theorems 4.1-4.5 hold, then (1.1), (1.2) has a unique steady state.

5. Concluding remarks. A sequence of nested, globally finitely attracting, invariant regions of the phase space of (1.1), (1.2), (2.1), converging to an invariant, globally asymptotically stable region, has been obtained in $\$ 3$. In $\$ 4$, some quantitative sufficient conditions ( $\phi^{2}$ sufficiently large or small, or $g$ increasing and $\gamma$ sufficiently small) for global asymptotic stability of the steady state have been obtained, for a kinetic function $f$ of the type $f(u, v)=g(u) \exp (\gamma-\gamma / v)$. Some of the results, which were not uniformly valid in $0<\lambda<\infty$ if $\gamma$ is too large, have been explained by comparison with linear stability results that were obtained in [2]. Of course, similar results to those of $\& 4$ may be obtained for any kinetic function satisfying assumption (H.2), such as that in (1.6).

The results of $\$ 3$ remain valid when the Robin type of boundary data is replaced by Dirichlet boundary data $(u=1$ on $\partial \Omega)$, and $\sigma \int_{\partial \Omega}(1-u) d s$ is replaced by $\int_{\partial \Omega}(\partial u / \partial n) d s$ everywhere. To see that, a unit order (see, e.g., Amann [26]) must be used to replace the definition of the order relation $\ll$ at the end of $\$ 1$ by $u_{1} \ll u_{2}$ means that there is a positive constant $c$ such that $u_{1}(x)+c e(x) \leqq u_{2}(x)$ for all $x \in \Omega$, where the unit $e$ is defined by $\Delta e+1=0$ in $\Omega, e=0$ on $\partial \Omega$. Such order definition could have been used in $\S 3$ to obtain results for both Robin and Dirichlet problems at the same time, although it has not been done for the sake of clarity. 
Growth restrictions on the function $f$ are not necessary for the ideas of $\S 3$ to apply. The assumption $f_{v}(u, v)>0$ has been imposed because it is satisfied by the most commonly used kinetic functions (i.e., by those in (1.4)-(1.6)), but it could be removed; then the definition of the sequence (3.1)-(3.7) should be changed somewhat.

The ideas of this paper are naturally extended if: (a) the Laplacian operator $\Delta$ is replaced by a uniformly strongly elliptic operator; (b) the function $f$ and/or the boundary data depend on the space variable $x$; or (c) the linear boundary conditions in (1.1) are replaced by appropriate nonlinear ones. They apply also to some more general reaction-diffusion problems, such as the nonisothermal model (1.1), (1.3) (this point is currently under research). Nevertheless, (1.1), (1.2) has been considered first because such isothermal model (a) has practical interest in itself (not only as a limit of (1.1), (1.3)), as was explained in the Introduction, and (b) it retains the main intrinsic difficulty of (1.1), (1.3), namely, the flow defined by (1.1), (1.2) is not monotone. Also, global stability results for (1.1)-(1.2) may be (and have been) compared with local stability results, which were obtained in [2] for the slab geometry and first-order Arrhenius kinetics.

Appendix. Let us consider the elliptic semilinear problem

$$
\Delta u=\Lambda g(u) \text { in } \Omega, \quad \partial u / \partial n=\sigma(1-u) \text { on } \partial \Omega,
$$

where $\Lambda \geq 0, \sigma>0, \Omega \subset \mathbf{R}^{p}\left(p=1,2\right.$, or 3) satisfies assumption $(\mathbf{H} .1)$ and the $C^{\prime}$ function $g$ satisfies (4.2).

LEMMA A.1. Under the assumptions above:

A. The problem (A.1) possesses a minimal and a maximal solution, $u, \tilde{u} \in C^{2}(\bar{\Omega})$ such that

$$
0 \ll \underline{u} \leqq \tilde{u} \ll 1 .
$$

B. The solution of (A.1) is unique if $0 \leqq \Lambda<\alpha / k_{1} \leqq \infty$, where $\alpha>0$ is the smallest eigenvalue of (2.2), and $k_{1}$ is given by (4.9). Furthermore, if $u_{1}$ and $u_{2}$ are the solutions of (A.1) for $\Lambda=\Lambda_{1}$ and for $\Lambda=\Lambda_{2}$ with $0 \leqq \Lambda_{2}<\Lambda_{1}<\alpha / k_{1} \leqq \infty$, then

$$
0 \ll u_{2}-u_{1} \leqq k_{2}\left[k_{3}+k_{4} \Lambda_{1} k_{1} /\left(\alpha-\Lambda_{1} k_{1}\right)\right]\left(\Lambda_{1}-\Lambda_{2}\right) \text {, }
$$

where

$$
k_{2}=\max \{g(u): 0 \leqq u \leqq 1\}, \quad k_{3}=\max \left\{\psi_{1}(x): x \in \bar{\Omega}\right\}, \quad k_{4}=\max \left\{\psi_{2}(x): x \in \bar{\Omega}\right\},
$$

$\psi_{1} \gg 0$ is the unique solution of

$$
\Delta \psi_{1}+1=0 \quad \text { in } \Omega, \quad \partial \psi_{1} / \partial n+\sigma \psi_{1}=0 \quad \text { on } \partial \Omega,
$$

and $\psi_{2}$ is any eigenfunction of (2.2) such that $\psi_{2} \geqq \psi_{1}$.

Proof. A. For the existence of the minimal and maximal solutions of (A.1) see, e.g., [13], [14], or [20]. Inequalities (A.2) follow by standard arguments, using maximum principles.

B. Since the function $u \rightarrow g(u)+k_{1} u$ is nondecreasing in $0 \leqq u \leqq 1, U=\tilde{u}-u$ satisfies

$$
\Delta U+\Lambda k_{1} U \geqq 0 \quad \text { in } \Omega, \quad \partial U / \partial n+\sigma U=0 \quad \text { on } \partial \Omega .
$$

Then, if $\Lambda k_{1}<\alpha$, the generalized maximum principle (see [17]) shows that $U \leqq 0$. Therefore, $y=\tilde{u}$ and the solution of (A.1) is unique.

Since the smallest eigenvalue of (2.2) depends continuously on the parameter $\sigma$ (see, e.g., [22]), one may choose $\varepsilon>0$ sufficiently small for the smallest eigenvalue of

$$
\Delta \psi+\alpha_{1} \psi=0 \quad \text { in } \Omega, \quad \partial \psi / \partial n+(\sigma-\varepsilon) \psi=0 \text { on } \partial \Omega,
$$


$\alpha_{1}$, to be such that $k_{1} \Lambda_{1} \leqq \alpha_{1}$. Then if $\psi \gg 0$ is an eigenfunction of (A.5), $U=\left(u_{2}-u_{1}\right) / \psi$ satisfies

$\psi \Delta U+2 \nabla \psi \cdot \nabla U \leqq \Lambda_{1}\left[g\left(u_{2}\right)-g\left(u_{1}\right)\right]+\alpha_{1}\left(u_{2}-u_{1}\right) \quad$ in $\Omega, \quad \partial U / \partial n+\varepsilon U=0 \quad$ on $\partial \Omega$.

Then, standard maximum principles show that $U \gg 0$, i.e., that $u_{2} \gg u_{1}$.

Finally, $U=u_{2}-u_{1}-\left(\Lambda_{1}-\Lambda_{2}\right) k_{2}\left[\psi_{1}+\Lambda_{1} k_{1} \psi_{2} /\left(\alpha-\Lambda_{1} k_{1}\right)\right]$ is easily seen to satisfy (A.4) with $\Lambda=\Lambda_{1}$. Therefore, $U \leqq 0$ and the second inequality (A.3) readily follows.

Remark. If $g^{\prime}(u) \geq 0$ for all $u \in[0,1]$, then $k_{1}=0$, the solution of (A.1) is unique for all $\Lambda \geq 0$ and inequalities (A.3) become $0 \ll u_{2}-u_{1} \leqq k_{2} k_{3}\left(\Lambda_{1}-\Lambda_{2}\right)$. Under an additional mild assumption on the function $g$, the following lemma provides another upper bound to $u_{2}-u_{1}$, which is stronger than that above when $\Lambda_{2}$ is large.

LemmA A.2. In addition to the assumptions of Lemma A.1, let us assume that

$$
k_{7}=\sup \left\{g(u) / u g^{\prime}(u): 0<u \leqq 1\right\}<\infty .
$$

Let $u_{1}$ and $u_{2}$ be the solutions of (A.1) for $\Lambda=\Lambda_{1}$ and $\Lambda=\Lambda_{2}$ with $0 \leqq \Lambda_{2}<\Lambda_{1}<\infty$. Then

$$
0 \ll u_{2}-u_{1} \leqq\left[1-\left(\Lambda_{2} / \Lambda_{1}\right)^{k_{7}}\right] \max \left\{u_{2}(x): x \in \bar{\Omega}\right\} \text {. }
$$

Remark. Assumption (A.6) implies that $g^{\prime}(u)>0$ for all $0<u \leqq 1$. Although the converse is not true in general, it is true if, for example, the function $u \rightarrow g^{\prime}(u)$ is nondecreasing in a neighborhood of $u=0$, as is the case for most commonly used kinetic functions (e.g., for those given in (1.4), (1.5)).

Proof. $U=u_{2}-u_{1} \gg 0$ satisfies

$$
\Delta U=\Lambda_{2} g\left(u_{2}\right)-\Lambda_{1} g\left(u_{1}\right) \quad \text { in } \Omega, \quad \partial U / \partial n+\sigma U=0 \quad \text { on } \partial \Omega .
$$

Let $x_{0}$ be a point (not necessarily unique) where the maximum of $U$ is attained. Since $\sigma>0$ and $U\left(x_{0}\right)>0, x_{0}$ cannot be a point of $\partial \Omega$. Then, $\Delta U \leqq 0$ at $x=x_{0}$ and

$$
\Lambda_{2} / \Lambda_{1} \leqq g\left(u_{1}\left(x_{0}\right)\right) / g\left(u_{2}\left(x_{0}\right)\right) \leqq\left[u_{1}\left(x_{0}\right) / u_{2}\left(x_{0}\right)\right]^{1 / k_{7}},
$$

where the second inequality is easily obtained when using (A.6) (the function $u \rightarrow$ $g(u) / u^{1 / k_{7}}$ is nondecreasing). Then the conclusion of the lemma readily follows.

Let us assume now that the function $g$ is such that there exists a constant $a$, $0<a \leqq 1$, satisfying

$$
\begin{gathered}
g^{\prime}(u)>0 \quad \text { for all } 0<u \leqq a, \quad g(a)<g(u) \text { for all } a<u \leqq 1, \\
k_{8}=\sup \left\{g(u) / u g^{\prime}(u): 0<u \leqq a\right\}<\infty .
\end{gathered}
$$

Then, we have the following.

LEMMA A.3. Let us assume that, in addition to the hypothesis of Lemma A.1, (A.7) holds. If

$$
\Lambda G(a) \geqq \sigma\left(\sigma+p / \rho_{1}\right)
$$

then (A.1) has a unique solution, $u=u(x)$, which satisfies

$$
\Lambda G(u(x)) \leqq \sigma\left(\sigma+p / \rho_{1}\right) \text { for all } x \in \bar{\Omega},
$$

where $\rho_{1}$ is defined in the interior sphere property (assumption (H.1); see Introduction), and $G:[0,1] \rightarrow \mathbf{R}$ is the strictly increasing function

$$
G(u)=\int_{0}^{u} g(z) d z
$$


If, in addition, (A.8) holds and if $u_{1}$ and $u_{2}$ are the solutions of (A.1) for $\Lambda=\Lambda_{1}$ and $\Lambda=\Lambda_{2}$, where $\Lambda_{1}$ and $\Lambda_{2}$ satisfy (A.9) and $\Lambda_{2}<\Lambda_{1}<\infty$, then

$$
0 \ll u_{2}-u_{1} \leqq\left[1-\left(\Lambda_{2} / \Lambda_{1}\right)^{k_{8}}\right] \max \left\{u_{2}(x) ; x \in \bar{\Omega}\right\} \text {. }
$$

Proof. Let $u=u(x)$ be a solution of (A.1) and let $x_{0}$ be a point (not necessarily unique) of $\bar{\Omega}$ where the maximum of $u, u_{M}=\max \{u(x): x \in \bar{\Omega}\}$, is attained. $x_{0} \in \partial \Omega$ because otherwise $\Delta u\left(x_{0}\right)>0$. Let $S_{1} \subset \Omega$ be the hypersphere, of radius $\rho_{1}$, that is tangent to $\partial \Omega$ at $x_{0}$. We consider the problem

$$
\Delta w=\Lambda g_{1}(w) \quad \text { in } S_{1}, \quad w=u_{M} \text { on } \partial S_{1},
$$

where the $C^{1}$-function $g_{1}:[0,1] \rightarrow \mathbf{R}$ is such that $g_{1}(u)=g(u)$ for $0 \leqq u \leqq a, g_{1}^{\prime}(u)>0$ for $a<u \leqq 1$. Problem (A.13) has a unique solution (Lemma A.1), which is spherically symmetric (Gidas et al. [27]), and given by

(A.14) $\quad r^{1-p} d\left[r^{p-1} d w / d r\right] / d r=d^{2} w / d r^{2}+(p-1) r^{-1} d w / d r=\Lambda g_{1}(w) \quad$ in $0<r<\rho_{1}$,

$$
d w / d r=0 \quad \text { at } r=0, \quad w=u_{M} \text { at } r=\rho_{1},
$$

where $r=\overline{x_{1} x}$ and $x_{1}$ is the center of $S_{1}$. Furthermore, the solution of (A.13) satisfies $w(x) \geqslant u(x)$ for all $x \in S_{1}$, as it is easily seen by means of maximum principles. Hence

$$
\sigma\left(1-u_{M}\right)=(\partial u / \partial n)_{x=x_{0}} \geqq(d w / d r)_{r=\rho_{i}} \cdot
$$

On the other hand, integration of (A.14), (A.15) yields

$$
r^{p-1} d w / d r=\Lambda \int_{0}^{r} z^{p-1} g_{1}(w(z)) d z
$$

Therefore, the function $r \rightarrow w(r)$ is strictly increasing and (A.17) yields $p d w / d r<$ $\Lambda g_{1}(w(r))$ for all $0<r \leqq \rho_{1}$. Hence, (A.14) implies that the function $r \rightarrow d w / d r$ is also strictly increasing, and (A.17) leads to

$$
\begin{aligned}
\rho_{1}^{p-1}(d w / d r)_{r=\rho_{1}}^{2}>\Lambda \int_{\varepsilon \rho_{1}}^{\rho_{1}} z^{p-1} g_{1}(w(z))(d w / d z) d z \\
>\Lambda\left(\varepsilon \rho_{\mathrm{t}}\right)^{p-1}\left[G_{1}\left(u_{M}\right)-G_{1}\left(w\left(\varepsilon \rho_{\mathrm{l}}\right)\right)\right]
\end{aligned}
$$

for any real constant $\varepsilon$ such that $0<\varepsilon<1$, where

$$
G_{1}(u)=\int_{0}^{u} g_{1}(z) d z
$$

But, as it is seen from (A.17), (A.19),

(A.20) $\rho_{1}^{p-1}(d w / d r)_{r=\rho_{1}}>\Lambda g_{1}\left(w\left(\varepsilon \rho_{1}\right)\right) \int_{\varepsilon \rho_{1}}^{\rho_{1}} z^{p-1} d z=\Lambda g_{1}\left(w\left(\varepsilon \rho_{1}\right)\right) \rho_{1}^{p}\left(1-\varepsilon^{p}\right) / p$,

$$
G_{1}\left(w\left(\varepsilon \rho_{1}\right)\right)<w\left(\varepsilon \rho_{1}\right) g_{1}\left(w\left(\varepsilon \rho_{1}\right)\right)<u_{M} g_{1}\left(w\left(\varepsilon \rho_{1}\right)\right) .
$$

Equations (A.16), (A.18), (A.20)-(A.21) lead to the inequality

$$
\Lambda G_{1}\left(u_{M}\right)<\sigma\left(1-u_{M}\right)\left[\dot{\varepsilon}^{1-p} \sigma\left(1-u_{M}\right)+p u_{M} / \rho_{1}\left(1-\varepsilon^{p}\right)\right]
$$

which is valid for $0<\varepsilon<1$. Then, when replacing $\varepsilon^{p}$ by $1-u_{M}$, we obtain (recall that $0<u_{M}<1$ )

$$
\Lambda G_{1}\left(u_{M}\right)<\sigma\left(\sigma+p / \rho_{1}\right) .
$$

Since the function $G_{1}$ is strictly increasing and $G_{1}(u)=G(u)$ for $0 \leqq u \leqq a$, if $\Lambda$ satisfies (A.9), then (A.22) yields $u_{M}<a$, i.e., any solution of (A.1) satisfies

$$
u(x)<a \text { for all } x \in \bar{\Omega} \text {. }
$$


Then, (A.1) has a unique solution, as it comes out when Lemma A.1 and maximum principles are applied and (A.7) and (A.23) are taken into account. Inequality (A.10) is readily obtained from (A.22).

Finally, (A.12) is obtained by the argument of the proof of Lemma A.2, when taking into account that $u_{1}$ and $u_{2}$ satisfy (A.23).

LEMMA A.4. In addition to the assumptions of Lemma A.1, let us assume that $g^{\prime}(u)>0$ for all $0<u \leqq 1$, and let $u=u(x)$ be the (unique) solution of (A.1) for a given value of $\Lambda>0$. Then, $\left.u_{M}=\max \{u(x): x \in \partial \Omega\}=\max \{u(x): x \in \bar{\Omega})\right\}$, and $u_{m}=$ $\min \{u(x): x \in \partial \Omega\}$ satisfy

$$
\begin{gathered}
\sigma\left(1-u_{M}\right)>\Lambda G\left(u_{M}\right) /\left[p / \rho_{1}+\sqrt{\left(p / \rho_{1}\right)^{2}+2^{1-1 / p} \Lambda G\left(u_{M}\right)}\right] \\
\sigma\left(1-u_{m}\right)<\left(1+D / 2 \rho_{2}\right)^{p-1} \sqrt{2 \Lambda G\left(u_{m}\right)},
\end{gathered}
$$

where $\rho_{1}$ and $\rho_{2}$ are defined in the interior and exterior sphere properties (assumption (H.1); see Introduction), $D$ is the diameter of $\Omega$ and the strictly increasing function $G:[0,1] \rightarrow \mathbf{R}$ is defined by (A.11).

Proof. The argument that led to (A.21) in the proof of Lemma A.3 shows that $u_{M}$ satisfies

$$
\Lambda G\left(u_{M}\right)<\sigma\left(1-u_{M}\right)\left[\varepsilon^{1-p} \sigma\left(1-u_{M}\right)+p u_{M} / \rho_{1}\left(1-\varepsilon^{p}\right)\right]
$$

for all $0<\varepsilon<1$. Then, if $\varepsilon^{p}=\frac{1}{2}$ (A.24) is readily obtained.

Let $x_{0} \in \partial \Omega$ be a point (not necessarily unique) where $u_{m}$ is attained. Let $S_{2}$ be the hypersphere of radius $\rho_{2}$, tangent to $\partial \Omega$ at $x_{0}$ to which the exterior sphere property refers. Let $x_{2}$ be the center of $S_{2}$ and let $S$ be the hypersphere of center at $x_{2}$ radius $\rho_{2}+D$. Then $\Omega \subset S-\bar{S}_{2}$. Let $w: \bar{S}-S_{2} \rightarrow \mathbf{R}$ be defined by

$$
\Delta w=\Lambda g(w) \quad \text { in } S-\vec{S}_{2}, \quad w=u_{m} \text { on } \partial\left(S-\bar{S}_{2}\right) \text {. }
$$

Problem (A.26) possesses a unique solution, which is spherically symmetric, and given by

$$
\begin{gathered}
r^{1-p} d\left[r^{p-1} d w / d r\right] / d r=\Lambda g(w) \quad \text { in } \rho_{2}<r<\rho_{2}+D, \\
w=u_{m} \quad \text { at } r=\rho_{2} \text { and } r=\rho_{2}+D
\end{gathered}
$$

where $r=\overline{x_{2} x}$. To see this, observe that (A.27) has (at least) a solution (see, e.g., Keller [13]), and that (A.26) has (at most) one solution (Lemma A.J).

The (unique) solution of (A.26) satisfies (apply maximum principles)

$$
0<w(x)<u_{m} \text { for all } x \in S-\bar{S}_{2}, \quad w(x) \leqq u(x) \text { for all } x \in \bar{\Omega} .
$$

Hence

$$
-(d w / d r)_{r-\rho_{2}} \geqq(\partial u / \partial n)_{x=x_{0}}=\sigma\left(1-u_{m}\right) .
$$

On the other hand, let $r_{1}>\rho_{2}$ be the smallest value of $r$ where $d w / d r=0$. Since $r_{1} \leqq \rho_{2}+D / 2$ (see Gidas et al. [27]), when (A.27) is multiplied by $r^{2 p-2} d w / d r$ and the resulting equation is integrated between $\rho_{2}$ and $r_{1}$, we obtain

$$
\begin{aligned}
{\left[\rho_{2}^{p^{-t}}(d w / d r)_{r=\rho_{2}}\right]^{2} } & =-2 \Lambda \int_{\rho_{2}}^{r_{1}} r^{2 p-2} g(w(r))(d w / d r) d r \\
& \leqq 2 \Lambda r_{1}^{2 p-2} \int_{w_{1}}^{u_{m}} g(w) d w<2 \Lambda\left(\rho_{2}+D / 2\right)^{2 p-2} G\left(u_{m}\right)
\end{aligned}
$$

where $w_{1}=w\left(r_{1}\right)>0$. Then (A.25) readily follows from (A.28), (A.29), taking into account that $(d w / d r)_{r=\rho_{2}}<0$. 


\section{REFERENCES}

[1] R. ARis, The Mathematical Theory of Diffusion and Reaction in Permeable Catalysts, Vol. I and II, Clarendon Press, Oxford, 1975.

[2] I. E. PARRA AND J. M. VEGA, Local nonlinear stability of the steady state in an isothermal cotalyst, SIAM J. Appl. Math., 48 (1988), to appear.

[3] C. J. Pereira AND A. VARMA, Uniqueness criteria of the steady state in automotive catalysis, Chem. Engrg. Sci, 33 (1978), pp. 1645-1657.

[4] H. AMANN, Existence and stability of solutions for semilinear parabolic systems, and applicafions to sonte diffusion-reaction equations, Proc. Roy. Soc. Edinburgh, 81 A (1978), pp. 35-47.

[5] C. V. PAO, Asymptotic stability of reaction-diffusion systems in chemical reactor and combustion theory, J. Math. Anal. Appl., 82 (1981), pp. 503-526.

[6] J. HERNÁNDEZ, Some existence and stability results for solutions of reaction-diffusion systems with nonlinear boundary condisions, in Nonlinear Differential Equations: Invariance Stability and Bifurcation, P. de Mottoni, ed., Academic Press, New York, 1981.

[7] A. LEUNG AND D. CLARK, Bifurcations and large-fime asymptotic behavior for prey-predator reactiondiffusion equations with Dirichlet boundary data, J. Differential Equations, 35 (1980) pp. 113-127.

[8] C. V. PAO, On nonlinear reaction-diffusion systems, J. Math. Anal. Appl., 87 (1982), pp. 165-198.

[9] —_, Asymptotic stability of a coupled diffusion system arising from gas-liquid reactions, Rocky Mountain J. Math., 12 (1982), pp. 55-73.

[10] G. S. LADDE, V. LAKShMikANThAM, AND A. S. VATSAla, Existence and asymptotic behavior of reaction-diffusion syssems via coupled quasi-solutions, in Nonlinear Analysis and Applications, V. Lakshmikantham, ed., Academic Press, New York, 1982.

[1]] M. W. HiRSch, The dynamical systems approach to differential equations, Bull. Amer. Math. Soc, 11 (1984), pp. 1-64.

[12] A. LEUNG, Monotone schemes for semilinear elliptic systems related to ecology, Math. Meth. Appl. Sci., 4 (1982), pp. 272-285.

[13] H. B. KELLER, Eltiptic boundary value problems suggested by nonlinear diffusion processes, Arch. Rational Mech. Anal., 35 (1969), pp. 363-381.

[14] D. H. SAtTINGER, Monotone methods in nonlinear elliptic and parabolic boundary value problems, Indiana Univ. Math. J., 21 (1972), pp. 979-1000.

[15] J. C. Burnell, A. A. LACEy, AND G. C. WAKE, Steady states of the reaction-diffusion equations. Part 1: Questions of existence and continuity of solution branches, J. Austral. Math. Soc. Ser. B, 24 (1983), pp. 374-391.

[16] _-_ Steady states of the reaction-diffusion equations. Part II: Uniqueness of solutions and some special cases, J. Austral. Math. Soc. Ser. B, 24 (1983), pp. 392-416.

[17] M. H. Protter and H. F, Weinderger, Maximum Principles in Differential Equations, Prentice-Hall, Englewood Clifts, NJ. 1967.

[18] D. HENRY, Geometric theory of semilinear parabolic equations, in Lecture Notes in Math. 840, SpringerVerlag, New York-Berlin, 1981.

[19] H. AMANN, Periodic solutions of semilinear parabolic equations, in Nonlinear Analysis: A Coliection of Papers in Honour of Erich $\mathbf{H}$. Rothe, L. Cesary, R. Kannan, and H. F. Weinberger, eds., Academic Press, New York, 1978.

[20] J. SMOLler, Shock Waves and Reaction-Diffusion Equations, Springer-Verlag, New York-Berlin, 1983.

[21] W. H. RAY AND S. P. HASTINGS, The influence of the Lewis number on the dynamics of chemically reacting systems, Chem. Engrg. Sci., 35 (1980), pp. 589-595.

[22] R. Courant AND D. Hilbert, Methods of Mathematical Physics, Vol. I, Wiley-Interscience, New York, 1953.

[23] E. N. DANCER, On the stracture of solutions of an equation in catalysis theory when a parameter is large, J. Differential Equations, 37 (1980), pp. 404-437.

[24] A. CASTRO AND R. SHIVAJI, Uniqueness of positive solutions for a class of elliptic boundary valut problems, Proc. Roy. Soc. Edinburgh, 98A (1984), pp. 267-269.

[25] R. ShivaJi, Remarks on an S-shaped bifurcation curve, J. Math. Anal. Appl., to appear.

[26] H. AMANN, Fixed point equations and nonlinear eigenvalue problems in ordered Banach spaces, SIAM Rev., 18 (1976), pp. 620-708.

[27] B. GIDAS, W. NI, AND L. NIRENBERG, Symmetry and related properties via the maximum principle Comm. Math. Phys., 68 (1979), pp. $209-243$. 